EUROPEAN ORGANIZATION FOR NUCLEAR RESEARCH

European Laboratory for Particle Physics

CERN - SL - PPE DIVISION

CERN-SL/97-40 (EA)

\title{
Study of Beam-Induced Particle Backgrounds at the LEP Detectors
}

\author{
G. von Holtey ${ }^{a}$, A. H. Ball ${ }^{b}$, E. Brambilla ${ }^{c}$, H. Burkhardt ${ }^{a}$, \\ P. Estabrooks ${ }^{d}$, F. J. Harris ${ }^{e}$, G. Lutters ${ }^{a}$, J. Rothberg ${ }^{f}$, \\ P. Roudeau ${ }^{g}$, P. Siegrist ${ }^{a, h}$, J. Wear ${ }^{i}$, P. Weber ${ }^{j}$, E. Vallazza $^{k}$
}

\begin{abstract}
Synchrotron radiation photons and off-energy electrons which contribute to backgrounds in the LEP experiments have been simulated. A multi-faceted background protection system has been designed and installed which permits the experiments to take data under very clean conditions. The backgrounds have been measured at LEP1 and are in good agreement with calculations. These simulations and studies have led to a design of enhanced background protection for LEP2 where the photon background is expected to be far more severe. Detector sensitivity to backgrounds and monitor systems are described.
\end{abstract}

Geneva, Switzerland

June 30, 1997

To be submitted to Nuclear Instruments and Methods 
${ }^{a}$ European Laboratory for Particle Physics (CERN), CH-1211 Geneva 23, Switzerland

${ }^{b}$ University of Maryland, Physics Dept. College Park, Maryland 20742 USA

${ }^{c}$ Massachusetts Institute of Technology, Cambridge, MA 02139, USA

${ }^{d}$ Carleton University, Dept. of Physics, Colonel By Drive OTTAWA, Ontario, Canada K1S 5B6

${ }^{e}$ Department of Physics, University of Oxford, Keble Road, Oxford OX1 3RH, UK

${ }^{f}$ Experimental Elementary Particle Physics, University of Washington, Seattle WA 98195, U.S.A.

${ }^{g}$ Université de Paris-Sud, Lab. de l'Accélérateur Linéaire, IN2P3-CNRS, Bât. 200, F-91405 Orsay Cedex, France

${ }^{h}$ DAPNIA/Service de Physique des Particules, CE-Saclay, F-91191 Gif-sur-Yvette Cedex, France

${ }^{i}$ Department of Radiology, University of Pennsylvania, Philadelphia, Pennsylvania, 19104, USA

${ }^{j}$ Grinnell College, Grinnell, Iowa 50112, USA

${ }^{k}$ Dipartimento di Fisica, Università di Trieste and INFN, Via A. Valerio 2, I-34127

Trieste, Italy 


\section{Contents}

1 Introduction 5

2 Background Sources and Protection System 6

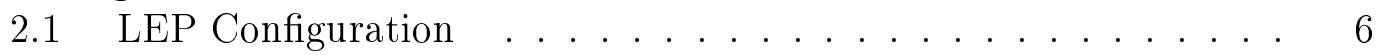

2.1.1 LEP Cycle . . . . . . . . . . . . . 8

2.1.2 Bunch Configurations ............. 8

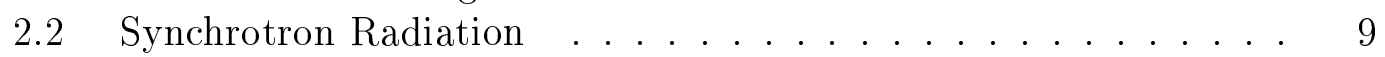

2.2.1 Photon Spectrum . . . . . . . . . . . . . . . . 9

2.2.2 Photon Background Simulation . . . . . . . . . 11

2.3 Off-Energy Particles and Beam Lifetime . . . . . . . . . . . . . 12

2.3.1 Loss Mechanisms . . . . . . . . . . . . . 12

2.4 Background Protection . . . . . . . . . . . . . 15

2.4.1 Background protection for LEP1 . . . . . . . . . 17

2.4.2 Improved Background protection for LEP2 . . . . . . . 20

3 Backgrounds in the Experimental Detectors 25

3.1 Vacuum Chamber ................ 27

3.2 Silicon Vertex Detectors . . . . . . . . . . . . 27

3.3 Gas Ionization Detectors . . . . . . . . . . . 28

3.3.1 The Time Projection Chamber . . . . . . . . 29

3.3.2 Wire Detector Aging .............. 29

3.4 Luminosity Calorimeters . . . . . . . . . . . . . 30

3.5 Background Monitoring . . . . . . . . . . . 30

3.5.1 Photon Rate Monitor . . . . . . . . . . . . . 31

3.5.2 Electron Rate Monitor ............. . 31

3.5.3 Multi-purpose Background Monitors . . . . . . . . . 31

3.5.4 Photon Arrival Time .............. 32

3.5.5 Radiation Monitor ............... 32

3.5.6 Beam Dump . . . . . . . . . . . 32

3.6 Background Simulations in the Experimental Detectors . . . . . . 34

3.6.1 Estimate of Photon Rates . . . . . . . . . . . . 35

4 Background Measurements 36

4.1 Aperture Limits . . . . . . . . . . . . . . . 36

4.2 Off-energy background . . . . . . . . . . . . . . . . . 36

4.2.1 Origin and Consequences . . . . . . . . . . . 36

4.2.2 Summary of observations . . . . . . . . . . . 37

4.2.3 Effect of off-energy electrons on luminosity measurements . 37

4.3 Arc Dipole Photons . . . . . . . . . . . . . . . . . 37

4.3.1 Evidence for Arc Dipole Photons . . . . . . . . . 39

4.4 Bunch Trains . . . . . . . . . . . . . . . . . . 40 
4.4.1 Bunch trains with horizontal crossing angles . . . . . . . 40

4.4.2 Bunch trains with head-on collisions . . . . . . . . . . 42

4.4.3 Off-energy electron background with bunch trains. . . . . . 46

4.5 Tests of the Synchrotron Radiation Masks . . . . . . . . . . . 48

4.5.1 The Background Measurements ............ 49

4.5.2 Comparison with Monte Carlo Simulations . . . . . . 50

$5 \quad$ Expectations at High Energy 53

5.1 LEP2 Parameters . . . . . . . . . . . . . . . 53

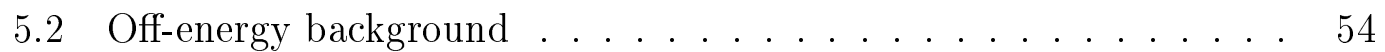

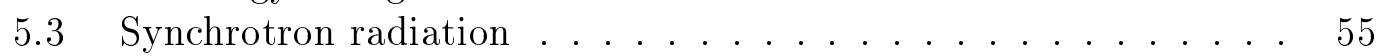

$\begin{array}{lll}6 \text { Conclusion } & 58\end{array}$

A Collimators and Masks $\quad 64$

B Detector Properties $\quad 66$ 


\section{$1 \quad$ Introduction}

The Large Electron-Positron Collider (LEP) is situated at CERN (European Laboratory for Particle Physics) in Geneva, Switzerland. Counter-rotating beams of electrons and positrons at energies from $45 \mathrm{GeV}$ to $96 \mathrm{GeV}$ collide at four Interaction Points equally spaced around the $27 \mathrm{~km}$ ring. At each interaction point a large scale general purpose particle detector system is used to record the products of electron-positron collisions. These detectors all include the capability for charged particle tracking with high precision as well as calorimetric devices for energy measurements.

The four physics experiments have been operational at LEP since 1989, running at about $45 \mathrm{GeV}$ per beam, and are expected to continue to take data until 2000. Starting in late 1995 periodic upgrades in energy will permit data taking up to $96 \mathrm{GeV}$ per beam.

Several important goals of these experiments, the search for the Higgs particle, the study of $B^{0} \bar{B}^{0}$ oscillations, precision measurements of $\tau$ lepton properties, require the identification of very short-lived particles ( $B$ mesons, $D$ mesons, $\tau$ leptons, etc) via their decay products. Since these particles have a mean decay length of typically 1-2 mm, detector elements must be placed very close to the beam vacuum pipe and must allow for extrapolation of individual tracks found within high multiplicity jets.

The innermost precision detectors use both silicon technology and gas ionization drift chambers. These detectors are sensitive to photons above $10 \mathrm{keV}$, as well as to charged particles, and are vulnerable to high levels of soft photon radiation. The effect of high radiation levels is either immediate destruction of portions of the detector or instability and long term degradation of performance.

The main particle backgrounds at LEP are off-energy electrons produced by beam-gas bremsstrahlung and synchrotron radiation photons produced in the closest bending magnets and in quadrupoles in the straight sections on either side of the experimental detectors. The off-energy electron rates under normal operation are acceptable and are not expected to increase with energy, except for effects due to increased dynamic pressure as a consequence of higher synchrotron radiation outgassing of vacuum components.

The synchrotron radiation background, on the other hand, even at $45 \mathrm{GeV}$, is many orders of magnitude larger than can be tolerated and must be reduced by strategic positioning of collimators and aperture limits. This background increases rapidly with beam energy and additional measures must be taken to permit detector operation at $96 \mathrm{GeV}$. It had been decided that installation of fixed masks in the vicinity of each detector could shadow the experiments from important sources of synchrotron radiation. An extensive series of background studies were undertaken at $45 \mathrm{GeV}$ to verify calculations and to permit a reliable extrapolation to higher energies. It was on the basis of these studies that the masks as well as new collimators and shielding systems were designed. 
This paper discusses the background sources and protection systems, the sensitivity of the detectors, background simulations, and studies of background conditions leading to the design of enhanced protection methods for higher energy beams.

\section{Background Sources and Protection System}

\subsection{LEP Configuration}

The Large Electron-Positron Collider (LEP) accelerates bunches of counter rotating positrons and electrons up to energies of $45 \mathrm{GeV}$ (LEP1), and eventually to $96 \mathrm{GeV}$ per beam (LEP2). The bunches collide at four diagonally opposite locations around the ring. Each of these interaction points (IP) is occupied by a LEP experiment: L3 in IP2, ALEPH in IP4, OPAL in IP6, and DELPHI in IP8.

The $27 \mathrm{~km}$ long LEP ring has an 8-fold symmetry, with each octant consisting of $\mathrm{a} \approx 500 \mathrm{~m}$ long straight section, centred around the IP, and $\approx 1.4 \mathrm{~km}$ long bending arcs on either side, (Fig. 1).

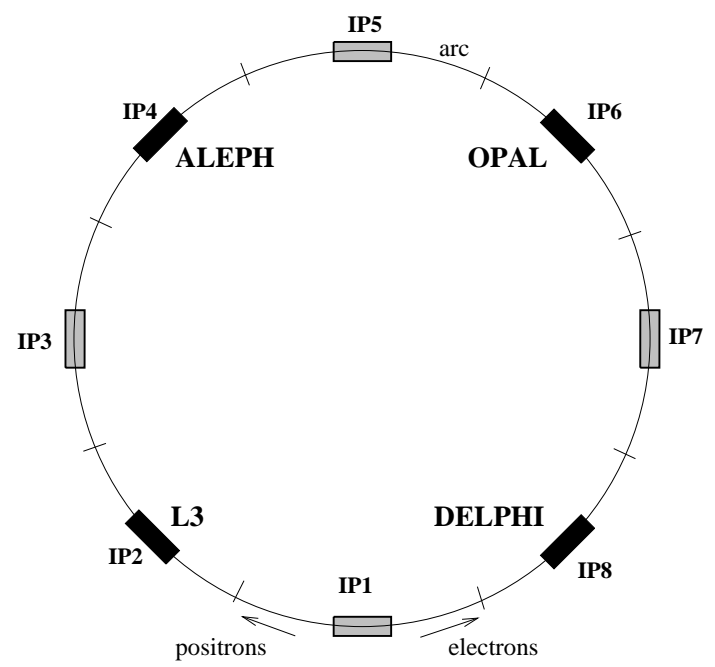

Figure 1: Schematic view of the LEP collider showing the eight-fold symmetry of the magnetic structure and the even numbered interaction regions that are occupied by the LEP experiments.

In order to reduce the energy and flux of synchrotron radiation photons that enter the straight sections and become a potential source of background radiation to the experiments, the field strength of the last bending dipole at the entrance to the straight sections is reduced to $10 \%$ of the normal arc dipole field. This weak bend radiation is shown in Fig. 2. 


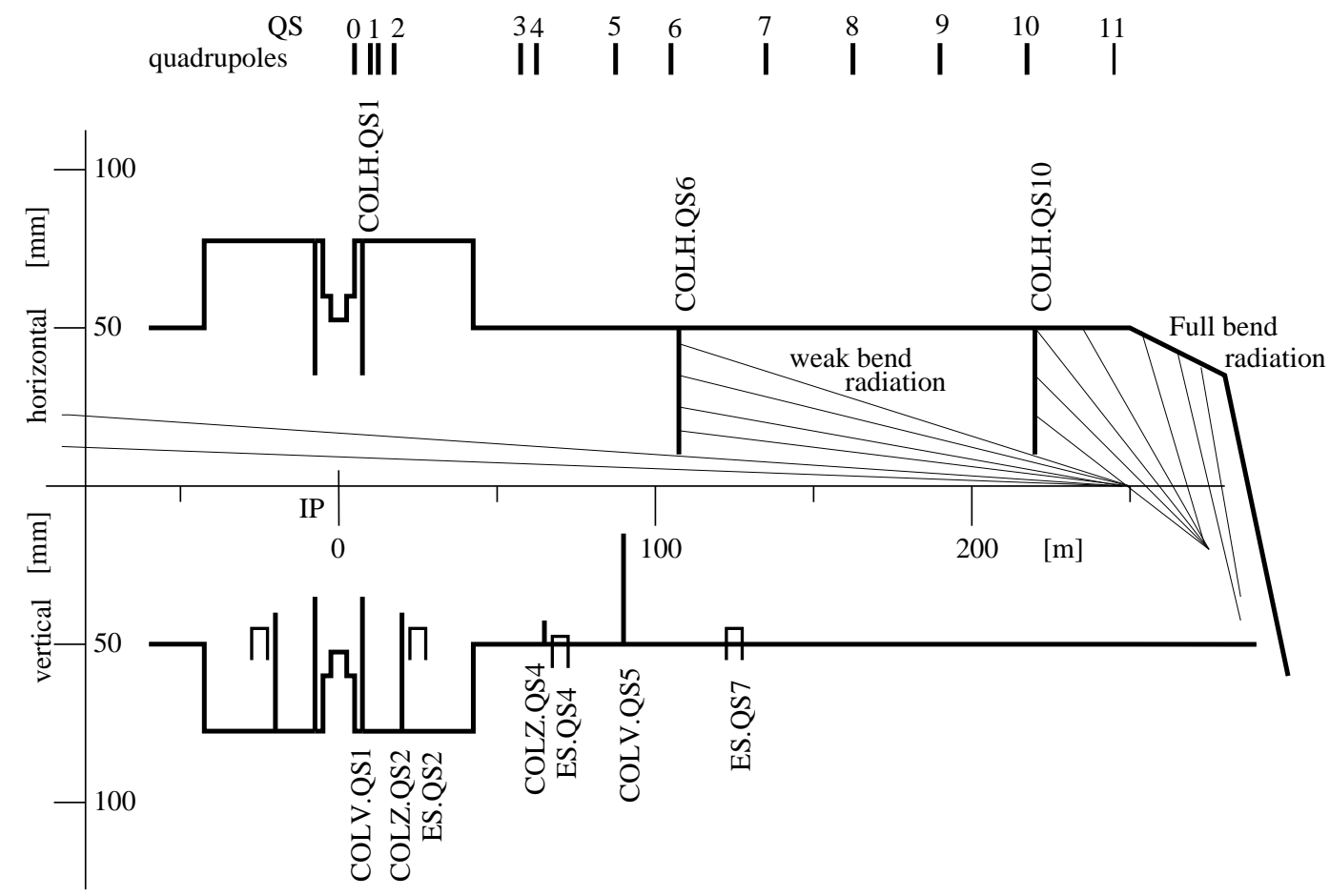

Figure 2: Layout of the straight section around IP4 or IP8 in the horizontal and vertical planes. Shown are the locations of quadrupoles (QS), electrostatic separators (ES) and collimators (COLH, COLV, COLZ). The solid lines mark the inner vacuum chamber radii for the LEP1 layout.

The odd numbered insertions contain specific beam equipment, such as beam instrumentation (IP1), wiggler magnets (IP3 and IP7), and the beam dump as well as a collimator system to artificially limit the machine apertures (IP5). Radiofrequency acceleration stations (RF) are concentrated on either side of IP2 and IP6. To reach LEP2 beam energies many more superconducting RF cavities are being installed, mainly at IP4 and IP8.

The bunches are focussed into the interaction regions by quadrupolar fields distributed along the straight sections (Fig. 2). Normalised field gradients of these quadrupoles lie in the range $k=0.02$ to $0.04 \mathrm{~m}^{-2}$, except for the strong superconducting low $\beta$ quadrupoles (QS0), adjacent to the even IP's, which have gradients of $k=0.16 \mathrm{~m}^{-2}$. The energy normalised gradient is defined by

$$
k=g / B \rho
$$

where $g[\mathrm{~T} / \mathrm{m}]$ is the gradient of the quadrupolar field and the beam rigidity $B \rho$ $[\mathrm{Tm}]=3.3356 E_{\text {beam }}[\mathrm{GeV} / \mathrm{c}]$. The transverse rms size of the beams at the IP are given by

$$
\sigma_{x, y}^{*}=\sqrt{\epsilon_{x, y} \beta_{x, y}^{*}}
$$


Table 1: Typical values of beam parameters at the interaction points.

\begin{tabular}{|cccc|}
\hline plane & $\epsilon[\mathrm{nm}]$ & $\beta^{*}[\mathrm{~m}]$ & $\sigma^{*}[\mu \mathrm{m}]$ \\
\hline$x$ & 35 & 2.5 & 296 \\
$y$ & 0.7 & 0.05 & 5.9 \\
\hline
\end{tabular}

Here $\epsilon_{x, y}$ are the transverse emittances of the beams and $\beta_{x, y}^{*}$ are the betatron functions at the IP in the horizontal $(x)$ and vertical $(y)$ planes, respectively. Typical values for beam emittances and rms beam sizes at the IP's for LEP1 at 45.6 GeV are given in Table 1.

In 1994 the quadrupole layouts in straight sections IP4 and IP8 were changed to create additional space between quadrupoles for installation of SC-cavities. As a consequence the machine optics in these two insertions is now different from the insertion optics in IP2 and IP6. As will be described later, this has important consequences for the amount of synchrotron radiation background seen in the different experiments.

\subsubsection{LEP CYCLE}

Electron and Positron bunches are injected at $22 \mathrm{GeV}$ from the SPS accelerator into LEP until beam currents of several $\mathrm{mA}$ per beam are accumulated. Both beams are then accelerated to $45.6 \mathrm{GeV}$, the energy required to produce a $\mathrm{Z}^{0}$. This is followed by a preparation phase where machine parameters are adjusted, the vertical $\beta$ is squeezed down to $5 \mathrm{~cm}$ (in the horizontal plane $\beta$ is $2.5 \mathrm{~m}$ ), and beams are brought into collision at the four interaction points. Finally, before stable beams for physics data-taking are declared, collimators are closed to predefined settings which protect the experiments from machine induced background particles. After a typically 12 hours (the luminosity lifetime is approximately 20 hours) the beams are dumped and a new cycle begins.

\subsubsection{Bunch Configurations}

On its way to higher luminosities, LEP has been running with different bunch configurations. The initial $4 \times e^{+}$on $4 \times e^{-}$bunch configuration was extended to an $8 \times e^{+}$on $8 \times e^{-}$bunch "Pretzel" configuration [1], where the unwanted additional bunch encounters at the mid-arc points were avoided by separating the beams through the arcs in the horizontal plane with electrostatic deflection fields. A further step was to introduce four counter-rotating trains of bunches. In this case unwanted bunch encounters take place on either side of the IP's. The effect of the additional beam-beam interactions due to the new encounters are controlled by separating the bunches on either side of the IP with orbit bumps. The bumps are built up by electrostatic separator fields and have, therefore, 
different orientation for the two oppositely charged beams. A first trial, using long separation bumps in the horizontal plane, thereby introducing horizontal crossing angles at the IP's, failed due to the very much enhanced photon background in the experiments from synchrotron radiation along the bumps [2]. Separating the bunch trains by short bumps in the vertical plane was more successful and is now used for physics data taking [3]. The same bunch train configuration, with two bunches per train, will be used for LEP2. Typical, good performance, beam characteristics for the three different LEP configurations are given in Table 2.

Table 2: Performance characteristics at $45.6 \mathrm{GeV}$ for different bunch configurations in LEP.

\begin{tabular}{|lcc|}
\hline LEP bunch configuration & $\begin{array}{c}\text { total current } \\
{[\mathrm{mA}]}\end{array}$ & $\begin{array}{c}\text { luminosity } \\
{\left[10^{31} \mathrm{~cm}^{-2} \mathrm{~s}^{-1}\right]}\end{array}$ \\
\hline $4 \times e^{+}$on $4 \times e^{-}$ & 3.4 & 10 \\
Pretzel $8 \times e^{+}$on $8 \times e^{-}$ & 5.5 & 20 \\
Bunch Trains $4 \times 3 e^{+}$on $4 \times 3 e^{-}$ & 8.2 & 20 \\
\hline
\end{tabular}

\subsection{Synchrotron Radiation}

In LEP the most serious beam-induced background to the experiments is from synchrotron radiation (SR) of electrons and positrons in quadrupole and dipole magnets. In spite of the measures taken in the general layout of the machine, with the experimental insertions in the centre of very long straight sections and a low field bending magnet at the end of the arcs (Fig. 2), the vacuum chambers of the high energy physics experiments would be struck by numbers of low energy photons, many orders of magnitude higher than can be tolerated by their detectors, unless additional carefully designed shielding were provided.

\subsubsection{Photon Spectrum}

The energy spectrum of SR photons radiated by a high energy electron travelling in a circular orbit of radius $\rho$ with $\gamma=E_{\text {beam }} / m_{0} c^{2}$, is characterised by a critical energy $E_{c}[5]$

$$
E_{c}=\frac{3}{2} \hbar c \gamma^{3} / \rho
$$

In LEP at $45.6 \mathrm{GeV}$ the Lorenz factor $\gamma=8.92 \times 10^{4}$, the bending radius $\rho=3096 \mathrm{~m}$, and the critical energy of the SR-spectrum from an arc dipole becomes $E_{c}=67.8 \mathrm{keV}$. Using the relative energy variable $q=E_{\gamma} / E_{c}$, the photon spectrum can be written independently of the photon energy $E_{\gamma}$, as

$$
d n / d q=\sqrt{3} \alpha \gamma I(q) \quad \text { with } \quad I(q)=\int_{q}^{\infty} K_{5 / 3}(q) d q
$$


where $\alpha$ is the fine-structure constant and $K_{5 / 3}$, a modified Bessel function of the third kind [5]. A typical photon spectrum from $45.6 \mathrm{GeV}$ electrons is shown in Fig. 3.

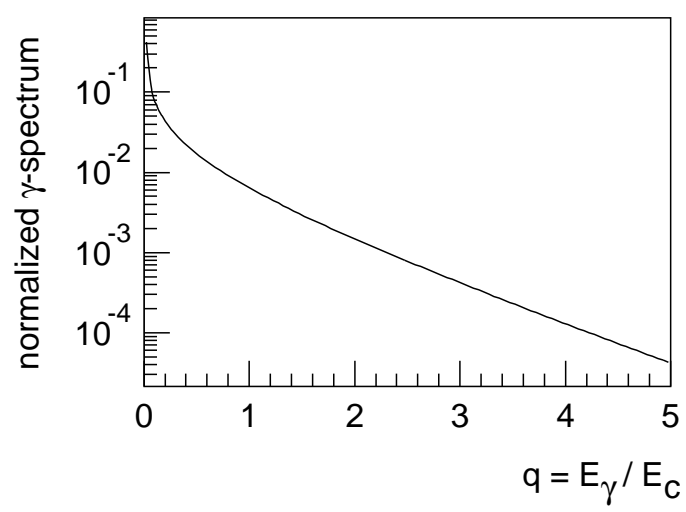

Figure 3: Number of synchrotron radiation photons as a function of the photon energy in units of the critical energy.

In quadrupolar fields, with normalized field gradient $k\left[\mathrm{~m}^{-2}\right]$, and betatron function $\beta$, an equivalent bending radius can be defined as

$$
(1 / \rho)_{\text {quad }}=k r^{*} \sqrt{\epsilon_{x} \beta_{x}+\epsilon_{y} \beta_{y}}
$$

where $r=\sqrt{x^{2}+y^{2}}$ is the distance of the electron orbit from the quadrupole axis, $\sigma_{r}=\sqrt{\epsilon_{x} \beta_{x}+\epsilon_{y} \beta_{y}}$ is the radial RMS beam size, and $r^{*}$ is the position of the orbit in the quadrupole in units of $\sigma_{r}$. While the energy spectrum from dipoles for a given machine depends only on the beam energy, the spectrum from quadrupole radiation depends also on the beam size. The photon spectrum radiated by a $45.6 \mathrm{GeV}$ electron on a $1 \sigma$ orbit $\left(\sigma_{x}=3.5 \mathrm{~mm}\right)$ in the low- $\beta$ quadrupole QS1 has a critical energy of $E_{c}=26.5 \mathrm{keV}$. At LEP2, with twice the beam energy and $40 \%$ larger emittances the critical energy will be eight times higher, $E_{c}=253 \mathrm{keV}$.

In LEP $\left(\gamma \approx 10^{5}\right)$ SR photons are radiated forward along the particle trajectory, within a small angular opening of $\pm 1 / \gamma$. The angular width of the cone of radiated photons from a quadrupole is therefore determined by the change of angle of the electron trajectory through the quadrupole. For LEP straight-section quadrupoles this quantity is of order $\sqrt{\epsilon_{x}}\left(x / \sigma_{x}\right)$ radians. Therefore photons radiated by particles in the beam tails $\left(x / \sigma_{x}>1\right)$ can shine on vacuum elements some $100 \mathrm{~m}$ downstream from the source and can reach the experiments directly, or after being forward or backward scattered from vacuum elements. The beam emittance ratio $\epsilon_{x} / \epsilon_{y}$ in LEP is much larger than 1 (see Table 1), therefore most photon background is generated in the horizontal plane, and increases with horizontal beam emittance. 
On average, every $45 \mathrm{GeV}$ electron radiates 0.5 photons with $E_{\gamma}>10 \mathrm{keV}$ per passage through the 26 straight-section quadrupoles, or 2.5 photons at a beam energy of $90 \mathrm{GeV}$. Without a complex system of collimators (see section 2.4), designed to shield the experimental detectors, far too many of these photons would strike the vacuum chamber in the experiments.

\subsubsection{Photon Background Simulation}

To optimise the layout for the collimator system, Monte Carlo simulation programs have been designed, that generate and track photons from all the relevant magnetic elements in the region of the experiments. Two independent Monte Carlo codes are used in LEP $[6,7]$. Electrons are generated out of the four dimensional transverse phase space and are tracked through the beam elements, where they radiate SR photons according to the spectrum of Eq. 4. Particular care is taken for a realistic simulation in the tails of the beam density distributions, as particles from the tails are most effective in producing background photons. SR photons with $E_{\gamma}>10 \mathrm{keV}$ are tracked until they hit the experimental chamber directly, or impinge on vacuum elements upstream or downstream of the IP, from where they can reach the detector after one scattering in the forward or backward direction. The photon reflection probability and energy loss are calculated from the incident photon energy and angle, taking into account the characteristics of the scattering material. Coherent and incoherent scattering and photon absorption have been included.

In particular for collimators close to the experiment, which tend to become important sources of back scattering, high $Z$ materials are clearly preferable [8]. The reflection probability of tungsten for SR photons in the backward direction is typically one order of magnitude smaller than for copper. However, heavy materials have the disadvantage of a very high fluorescence yield, and emit photons with relatively high energies (59.3 keV for tungsten). This makes it necessary to plate tungsten collimator surfaces with several layers of successively lighter materials, to absorb these fluorescent photons ${ }^{1}$. Fluorescence emission has not been included into the LEP simulation codes.

All simulations assume a perfectly aligned machine. Any misalignment of the beam with respect to the axis of the quadrupole magnet leads to radiation of more and higher energy photons as the beam passes through a higher magnetic field. It is further assumed that horizontal and vertical beam tails are limited with aperture collimators in the arcs, typically to less than $12.5 \sigma_{x}$ and $25 \sigma_{y}$ respectively, so that the presence of collimators upstream of an experiment at similar openings do not create electron background problems.

The described photon simulations in the LEP machine stop tracking photons once they have reached the beam pipe inside an experiment. In order to be able

\footnotetext{
${ }^{1}$ In the first generation of collimators for LEP 1 no surface plating has been included, however, the second generation collimators for LEP2 have silver and copper plated surfaces.
} 
to compare simulated photon rates with measured rates from the experiments, photons have to be further tracked, in a second step, into the different detectors. These simulations of backgrounds in the detectors are desribed in section 3.6. They result in defining detection probabilities for the different photon background families incident at the detector pipe which are seen in various background detectors.

In order to obtain the required statistical accuracy and to reach sufficient angular resolution, in particular for direct photon hits and for hits from forward scattering from inner collimator surfaces, the 19 two meter long quadrupoles have been divided into 20 slices each and a photon spectrum is radiated at the centre of each slice. For each run 20,000 electrons are tracked which radiate 7.6 million photons.

\subsection{Off-Energy Particles and Beam Lifetime}

The circulating beam particles in LEP can lose significant amounts of energy in scattering processes. As a result, the scattered particles can be lost and in some cases hit the detectors. This has been studied in detailed Monte Carlo simulation of the scattering processes and tracking through the magnetic lattice of LEP. Fig. 4 illustrates the tracking in LEP in the horizontal plane. The tracking is based on the Turtle Decay program [9]. Scattering angles are taken into account in the simulation, but are generally small compared to the beam divergence and deflection angles from energy loss in dispersion regions. The predicted background rates, energy and angular distributions agree with observations in the experiments and the total calculated loss rates match the observed beam lifetimes [10]. In this section we give an overview of the processes and some global results. Experimental results are given in section 4 .

Energy losses up to about $1 \%$ can be compensated by the radiofrequency acceleration system (RF bucket height). The horizontal aperture and magnet structure of LEP is such, that particles with up to about 3\% energy loss pass through the arcs. Beam particles with much larger energy losses can pass through the straight sections around the experiments. The fields of the quadrupole magnets in the straight sections, and in particular the strong superconducting low- $\beta$ quadrupoles next to the experiments, can bend particles with very substantial energy loss (more than 20\%) such that they enter the experimental detectors.

\subsubsection{LOSS MECHANISMS}

There are three main scattering processes that are almost entirely responsible for the losses of beam particles and therefore determine beam lifetimes in LEP:

i) Beam-beam Bremsstrahlung or (radiative) Bhabha scattering

ii) Compton scattering on thermal photons

iii) Beam gas scattering 


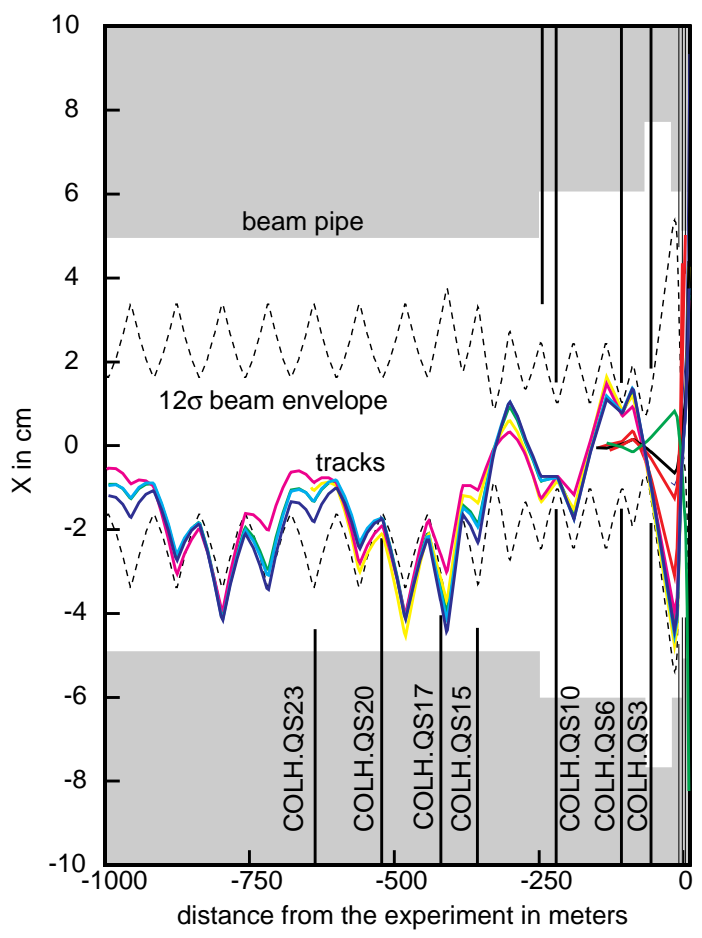

Figure 4: Illustration of beam particle tracking through the LEP lattice over 1000 meters up to an experimental region. The distance $X$ from the nominal orbit is given in cm units. The tracks are for particles that are lost within $\pm 9 \mathrm{~m}$ from the interaction point. The $12 \sigma$ beam envelope is shown as a broken line. The physical aperture limitation given by the beam pipes is shaded. The positions of collimators (called COLH.QS15, COLH.QS17...) as used in LEP physics runs are shown as vertical straight lines.

Their typical contribution to beam lifetimes in LEP1 is given in Table 3 . The

Table 3: Typical contributions to beam lifetimes in LEP1 in collisions

\begin{tabular}{|lcr|}
\hline B.B.-Bremsstrahlung & $\tau_{b}$ & $25 \mathrm{~h}$ \\
thermal photon & $\tau_{t}$ & $88 \mathrm{~h}$ \\
beam gas, $10^{-10}$ Torr & $\tau_{g}$ & $430 \mathrm{~h}$ \\
\hline total & $\tau$ & $19 \mathrm{~h}$ \\
\hline
\end{tabular}

beam-beam Bremsstrahlung from the collisions in the interaction regions is the dominant process for beam lifetime. A detailed Monte Carlo simulation for this process is described in [11]. The scattered particles leave the interaction region 
at very small angles and typically stay in the beam pipe for the next 100 meters, so that this process can be neglected as source of background to the experiments.

The second process, Compton scattering on the black body radiation in equilibrium with the walls of the beam pipe at room temperature, is only important for very high energy storage rings like LEP. In the context of storage rings, this process was first discussed by Telnov in 1987 [12]. The energy spectrum of the scattered photons has been measured in LEP and found to be in agreement with expectations [13]. The estimates used for this paper are based on the Monte Carlo program described in [14]. The energy spectrum of scattered photons, normalized to beam energy $E_{b}$, for LEP1 and LEP2 energies is shown in Fig. 5. The energy

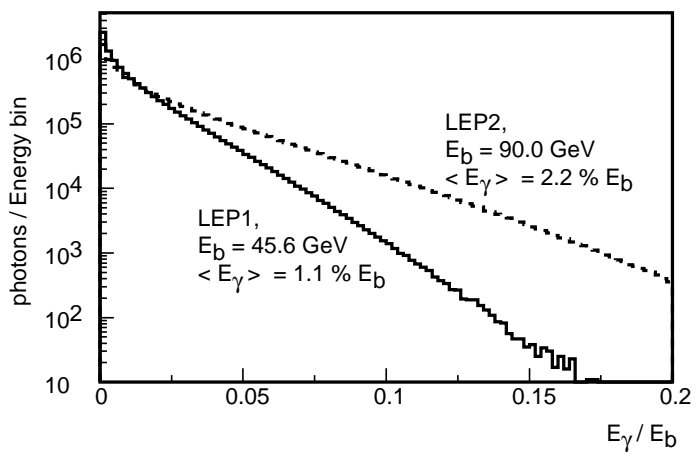

Figure 5: Energy spectrum of the scattered photons, normalized to beam energy for LEP1 and LEP2 energies.

of the initial thermal photon is on average $0.07 \mathrm{eV}$ which is very small compared with the beam energy. The energy of the scattered photon nearly equals the energy loss of the beam particle. The average energy of the scattered photons increases from $1.1 \%$ of the beam energy at LEP 1 to $2.2 \%$ for LEP 2 energies. The energy loss is too small to lead to off-energy particles being seen directly in the experiments.

Almost negligible for LEP1 beam lifetimes but most important as a source of off-energy particle background is the scattering of beam particles on residual gas atoms. The probability $P_{r}$ to radiate a photon with the energy fraction $k$ $\left(k=E_{\gamma} / E_{b}\right)$ per radiation length is approximately [15]:

$$
\frac{d P_{r}}{d k}=\frac{1}{k}\left(\frac{4}{3}-\frac{4}{3} k+k^{2}\right)
$$

General vacuum conditions in LEP are very good. The dynamic pressure rise in LEP dropped by nearly four decades during the first six years of LEP running and is now only a few $10^{-11}$ Torr/mA [16]. Estimates are usually based on CO-gas with a pressure of $10^{-10}$ Torr. For a given gas pressure, the variation of the radiation length $X_{0}$ with atomic number $\mathrm{Z}$ is roughly $X_{0} \propto \frac{1}{Z(Z+1)}$. Higher 
pressures, of the order of $10^{-9}$ Torr, are measured in sections equipped with normal conducting radiofrequency cavities. The broad energy spectrum present in the Bremsstrahlung process of beam-gas scattering, is the dominant source of off-energy beam particles with energy losses of more than $20 \%$. This can result in up to several hundred off-energy particles hitting an experimental detector per second.

Much shorter beam lifetimes than expected, and bursts of background to the experiments, have been observed for colliding beams and high beam currents, and have limited the maximum bunch currents at LEP1. They are attributed to non-gaussian tails in the transverse beam profiles, generated by the beam-beam effect.

Emittance blow-up is observed for colliding beams in LEP in both planes. A wiggler was used to artificially increase the horizontal beam sizes for both beams; this prevents uncontrolled flip-flop where one of the two beams blows up much more than the other. Non-gaussian tails have been studied quantitatively in LEP using collimators and loss monitors [17]. Very substantial tails have been observed in the vertical plane. A dependence on beam parameters like bunch currents or machine chromaticity-settings has been seen. For gaussian beam profiles, it is expected that collimators could be set to $7 \sigma$, without a measurable reduction in beam lifetime. The gaussian beam size is calculated according to:

$$
\sigma=\sqrt{\beta \epsilon+\left(D \frac{\Delta E}{E}\right)^{2}}
$$

with the global values of the beam emittance $\varepsilon$, and energy spread $\Delta E / E$, and the local values of the $\beta$-function and dispersion $\mathrm{D}[\mathrm{m}]$. The nominal emittances used to calculate the beam size were typically $45 \mathrm{~nm}$ for the horizontal and $4.5 \mathrm{~nm}$ for the vertical plane. These values correspond roughly to the largest emittances observed in routine operation. The energy spread $\Delta E / E$ is about $10^{-3}$.

Beam currents up to $4 \mathrm{~mA}$, or over $2 \times 10^{12}$ particles per beam, circulated in LEP1. A lifetime of 10 hours from scraping beam tails corresponds to $6 \times 10^{7}$ particles lost per second.

To reduce the probability of lifetime problems with colliding beams and nongaussian tails, we typically used collimator settings corresponding to $12 \sigma_{x}$ in the horizontal plane, and $25 \sigma_{y}$ in the vertical plane. These settings were used for the aperture collimators situated far from the experiments. To avoid scraping the beam close to the experiments, collimators around the interaction regions were opened by an additional $\approx 20 \%$.

\subsection{Background Protection}

Beam induced particle background rates in LEP are many orders of magnitude higher than can be tolerated by the LEP detectors [18]. Synchrotron radiation 
photons radiated in dipole and quadrupole fields can reach the experiments directly or after scattering from vacuum equipment along the $250 \mathrm{~m}$ straight sections on either side of the IP's. Off-energy particles created by beam-gas Bremsstrahlung reach the detectors after being over-focused in the strong low- $\beta$ quadrupoles. The energy spectra of the two background types are very different. While off-energy particles fill a broad spectrum centered around half the beam energy, synchrotron radiation photons have energies ranging between several keV to many hundred keV. Photons which come directly from synchrotron radiation in quadrupoles (direct photons) and those which are scattered before arrival (forward-scattered photons) have average energies of about $80 \mathrm{keV}$ for a $45 \mathrm{GeV}$ beam. Photons which are back scattered from obstacles downstream of the detector (backscattered photons) have lower energies, $\approx 50 \mathrm{keV}$, due to the inelastic Compton scattering process. Energy spectra for the different photon families at $45 \mathrm{GeV}$ and $90 \mathrm{GeV}$ are given in Fig. 6. While off-energy particles and direct and forward-scattered photons arrive in time with the beams, backscattered photons arrive at the detectors with a delay between $50 \mathrm{~ns}$ and $500 \mathrm{~ns}$, depending on the distance to the backscattering object.
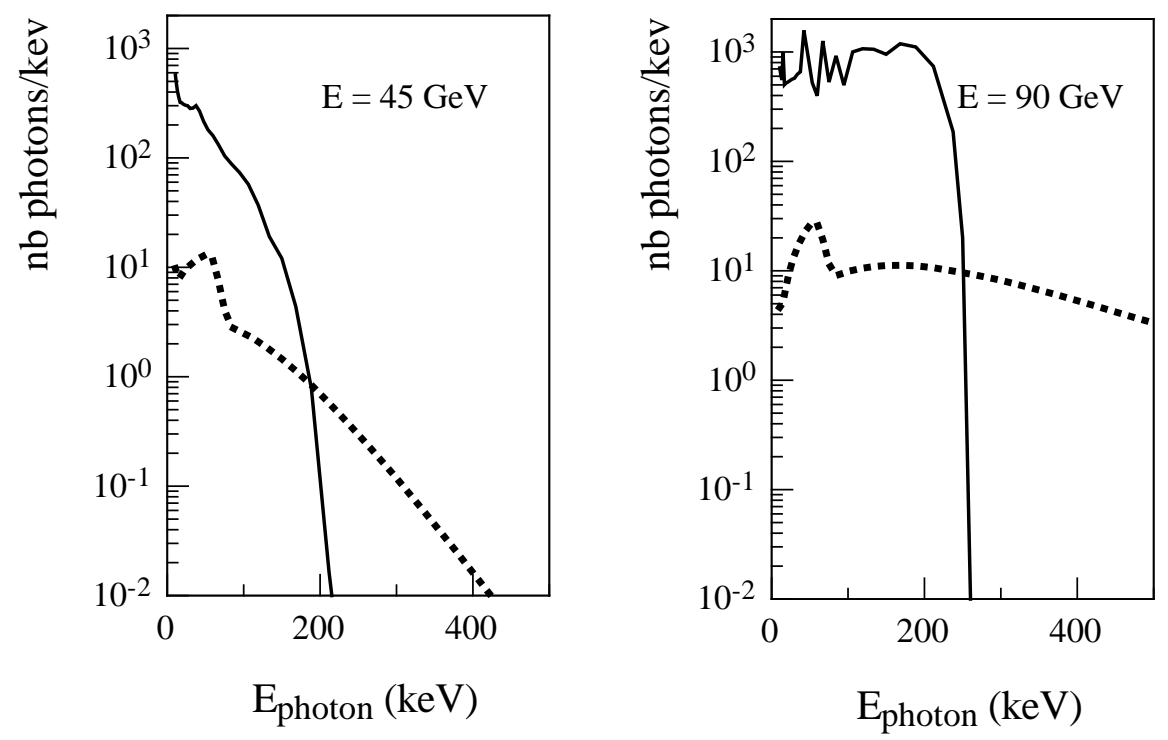

Figure 6: Energy spectra for forward (dotted lines) and backward (full lines) scattered background photons for two LEP beam energies. (left: $E_{\text {beam }}=45 \mathrm{GeV}$, $\epsilon_{x}=35 \mathrm{~nm}$; right: $\left.E_{\text {beam }}=90 \mathrm{GeV}, \epsilon_{x}=50 \mathrm{~nm}\right)$.

Synchrotron radiation photon and off-energy background sources in LEP have been extensively studied using Monte Carlo methods, in order to specify the collimator protection system to be included in the LEP design [8, 19]. Off- 
energy background particles can be simulated with great accuracy, but they are difficult to collimate as these particles stay close to the beam until the last strong quadrupoles, which bend them into the experiments. Simulation of synchrotron radiation photon background is much more complicated and consequently less accurate. On the other hand, photons can be collimated more easily. In LEP, without collimation, the experiments would be hit by approximately $10^{8}$ photons per $1 \mathrm{~mA}$ current in one bunch crossing $(\mathrm{BX} \times \mathrm{mA})$; seven orders of magnitude above tolerable levels.

\subsubsection{BACKGROUND PROTECTION FOR LEP1}

The LEP collimator protection system has developed over the years of LEP running and consists (1995) of 96 collimators (see Tables A.1 and A.2 in Appendix A). Of these, 90 are background collimators installed symmetrically around experimental insertions and six are aperture limiters located far from experiments in octant 5. Each collimator consists of one or two tungsten jaws of about 30 radiation lengths, with adjustable openings [20].

Two pairs of "far" collimators (Fig. 2), horizontal (QS6), and vertical(QS5), are placed at locations which, while removing all direct photons from the low-field bending magnets and most from the straight section quadrupoles, are sufficiently far away from the IP not to significantly increase the number of photons reflected into the experimental region. A second pair of "near" collimators, horizontal and vertical, are placed upstream of the second low- $\beta$ quadrupoles (QS1), $8.5 \mathrm{~m}$ from the IP. These collimators prevent all remaining direct photons from quadrupole radiation from hitting the vacuum chamber through the experiments (5.6 meters long and $106 \mathrm{~mm}$ inner diameter). They ensure, in combination with the far synchrotron radiation collimators, that the very high photon flux from the arcs cannot reach the detectors unless the photons are Compton scattered at least twice. The far collimators also reduce the number of photons radiated from distant quadrupoles which impinge on the jaws of near synchrotron radiation collimators and are scattered into the detectors.

The near synchrotron radiation collimators protect the experiments from scattered photons, reducing the photon rate at the beampipe by more than an order of magnitude. But the collimators themselves become scattering sources at reduced openings. The inner face of the upstream collimator jaw and the front face of the downstream tungsten jaw are intercepted by a rapidly increasing number of direct and scattered photons until they become, in spite of their reduced reflection probability, the dominating scattering source and limit the collimation efficiency. This is particularly true in the horizontal plane, because of the large emittance ratio in LEP, but becomes important also in the vertical plane when significant beam tails are present due to beam-beam effects.

Results of photon simulations at $45.6 \mathrm{GeV}$ of a simultaneous scan of both near collimators COLH.QS1, around IP4, predict a sharp minimum of the back- 


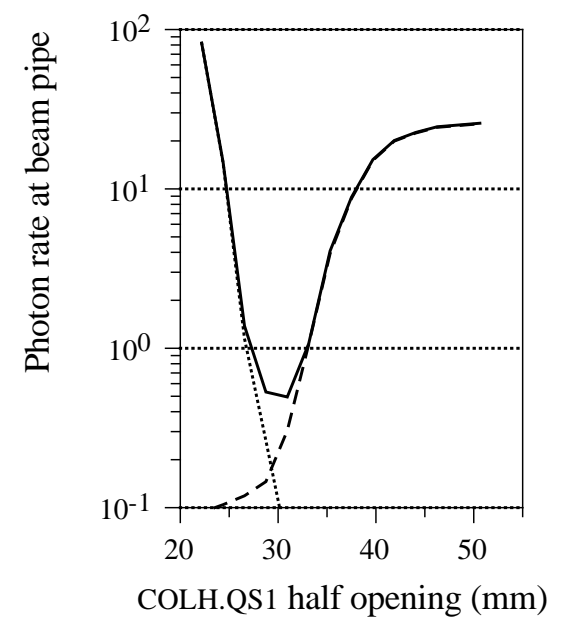

Figure 7: Simulated rate of synchrotron radiation photons incident on the beampipe in IP4 as function of the opening of the two "near" horizontal collimators COLH.QS1 in arbitrary units. Dotted line: forward plus backward scattered from COLH.QS1, broken line: backward scattered from downstream vacuum elements, full line: total photon rate from the horizontal and vertical plane. $\left(E_{\text {beam }}=45.6 \mathrm{GeV}, \epsilon_{x}=33 \mathrm{~nm}, \epsilon_{y}=2 \mathrm{~nm}\right.$, Gaussian beam density distributions)

ground rate at an opening of about $30 \mathrm{~mm}$ for beams with horizontal emittance of $\epsilon_{x}=33 \mathrm{~nm}$ (Fig. 7). At this optimum opening the reduced flux from backscattered photons from downstream beampipe elements, and the sharply increasing contribution of photons scattered from the COLH.QS1 collimator jaws, sum to produce a minimum. For the design of the LEP collimator protection system the longitudinal position of the near collimators with respect to the IP has been chosen to minimize the photon background level at the optimum opening. In practice the minimum is less pronounced, due to photons that reach the beampipe after multiple scatters, and also due to non-zero orbit deviations along the straight sections.

As the reflection probability for low energy photons from tungsten is 30 to 100 times larger in the forward direction than in the backward direction, up to half the rate from the near collimator jaws is due to forward scattering from the collimators intercepting the incoming beams. This contribution can be suppressed by inclining the inner jaw surfaces to be out of view for the incoming photon fans, an improvement incorporated in the second generation of horizontal near collimators for LEP2. The photon rate at large collimator openings in Fig. 7 is the sum of photons backscattered from different objects downstream of the IP. For openings below $40 \mathrm{~mm}$ it is dominated by photons back scattered from a region 
$50 \mathrm{~m}$ to $70 \mathrm{~m}$ from the IP, where the vacuum chamber diameter is reduced from $156 \mathrm{~mm}$ to $100 \mathrm{~mm}$ (see Fig. 2). At larger openings, photons back scattered from the far collimators, $90 \mathrm{~m}$ and $108 \mathrm{~m}$ from the IP, become important.

The opening of the near collimators for minimum photon background, as well as the level of this minimum, strongly depend on the horizontal beam size at the collimator, and therefore on the horizontal beam emittance and the amount of horizontal focusing at the interaction point. The latter is expressed by the horizontal betatron function $\beta_{x}^{*}$, at the IP. Large beam emittances, or strong focusing ( small $\beta_{x}^{*}$ ), increase the beam size at the collimator and force the optimum opening to larger values. At the same time the photon level at optimum opening is rapidly increased, due to a much larger number of backscattered photons from downstream vacuum elements for large horizontal beam emittances. For very small beam emittances, the optimum collimator opening cannot be reached if the collimator is not to cut into the machine aperture.

The described interdependence of photon background at the detectors, the horizontal beam emittance, and the focusing strength at the IP, was the basis for a proposal for a "background optics" in 1992, when large background rates and photon spikes were observed at the first part of physics fills with high bunch currents and very large horizontal emittances [21]. It was proposed to reduce the horizontal focusing by a factor of two, i.e. to increase $\beta_{x}^{*}$ from $1.25 \mathrm{~m}$ to $2.5 \mathrm{~m}$. As the LEP machine, with the high bunch currents reached, was beam-beam limited, the increased horizontal beam size at the IP's as consequence of the larger $\beta_{x}^{*}$ would not lead to a reduction of the luminosity. In 1993, running with the new "background optics", photon background rates at the LEP detectors were indeed much reduced.

The near SR collimators, at about $8.5 \mathrm{~m}$ from the IP, have the additional role of reducing off-energy electron backgrounds at the experiments. These particles originate from gas-bremsstrahlung in the arcs and straight sections. As shown in Fig. 8 only a weak protection of the central vacuum chamber is provided by the collimator with realistic openings of greater than $10 \sigma_{x}$. However, the rate of off-energy particles at the very forward luminosity detectors of the experiments can be substantially reduced, as these detectors are located downstream of the first low- $\beta$ quadrupoles.

The LEP1 background protection system (Table A.1 and A.2) also includes two pairs of vertical collimators, COLZ.QS2 and COLZ.QS4, primarily to protect electrostatic separator plates from synchrotron radiation photon impacts. In addition, two pairs of off-energy collimators, COLH.QS17 and COLH.QF23, located in the adjacent arcs, have the role of stopping off-energy particles that are produced in the arcs, from entering the straight sections. Two systems of aperture collimators, horizontal and vertical, are installed in octant 5, far from any experiment. Each system consists of three collimators, a primary collimator, and one secondary collimator for each beam. Secondary collimators are placed at a distance in betatron phase from the primary one that optimizes the proba- 


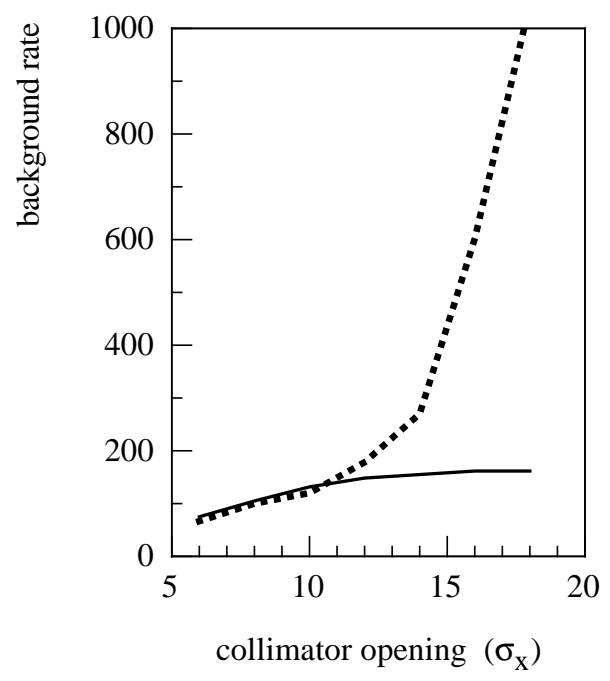

Figure 8: Simulated off-energy background rate as function of the near horizontal collimator opening. Background at central vacuum chamber (full line), background at luminosity detector at $8 \mathrm{~m}$ from IP within a band of radius $60 \mathrm{~mm}$ to $80 \mathrm{~mm}$ (dotted line).

bility of intercepting escaped particles from the primary collimator jaw. Detailed Monte Carlo studies [19], including tracking of high energy electrons through electromagnetic showers in the primary collimator blocks, have shown that the best collimation is obtained with high $Z$ material collimators with gently curved edge surfaces along the beam direction to make them insensitive to alignment errors. The escape probability for $50 \mathrm{GeV}$ electrons incident on a curved tungsten collimator jaw with impact distances of less than $100 \mu \mathrm{m}$ from the edge was found to be $5 \times 10^{-4}$. A two stage collimation allows this value to be reduced by an additional factor of 10 , with carefully placed secondary collimators.

\subsubsection{ImPROVED BACKGROUND PROTECTION FOR LEP2}

At a beam energy of $90 \mathrm{GeV}$ with $\mathrm{LEP}^{2}$ a much increased rate of synchrotron radiation photons incident on the beampipe must be expected, due to a large horizontal emittance of $50 \mathrm{~nm}$ and horizontal focusing of $\beta_{x}^{*}=1.25 \mathrm{~m}$. The "background optics" described above is not a solution for LEP2, as this machine will not be beam-beam limited and increasing $\beta_{x}^{*}$ would therefore lead to a substantial loss of potential luminosity. In fact, due to the increased beam energy

\footnotetext{
${ }^{2}$ This applies to the original optics version of LEP2 which used a horizontal phase advance per arc cell of $90^{\circ}$. An alternative version with $\Delta \mu=108^{\circ}$ phase advance per cell has also been considered. It has a nominal horizontal emittance of about $30 \mathrm{~nm}$ at $90 \mathrm{GeV}$.
} 
and the large beam emittance, the LEP1 background protection system can no longer cope with the expected high synchrotron radiation photon rates at LEP2 and must be improved [22].

Most of the additional photons at LEP2 come from backscattering sources $30 \mathrm{~m}$ to $70 \mathrm{~m}$ from the IP. In order to reduce this family, vacuum chambers in the region up to $55 \mathrm{~m}$ from the IP will be replaced by larger diameter chambers, including a tungsten absorber at their far end. Vacuum chambers through quadrupoles QS3 and QS4 will also be enlarged from their present $100 \mathrm{~mm}$ to a diameter of $156 \mathrm{~mm}$. Two pairs of horizontal synchrotron radiation collimators will be added: COLH.QS10 collimators to strengthen the role of the far synchrotron radiation collimators in intercepting the much increased photon flux from the arcs, and to protect super-conducting cavities against synchrotron radiation photons. Collimators COLH.QS3, located just downstream of the new cylindrical $156 \mathrm{~mm}$ bore tungsten absorber, will help to control the backscattered photon family, and will protect the new synchrotron radiation masks (see below) against direct photons from far quadrupoles. Both the photon absorber and the QS3 collimator have $\mathrm{Ag} / \mathrm{Cu}$ plated surfaces to reduce the emission of fluorescence photons from the tungsten absorbers. Absorber and collimator inner surfaces are inclined to avoid forward photon scattering.

Even with these improvements of the background protection system, the expected photon background rate under LEP2 conditions, is still about ten times higher than the rate at $45 \mathrm{GeV}$ for the same beam current [22]. As the beam currents at LEP2 are expected to at least double, the resulting photon rate remains much above acceptable levels. The remaining photon background is dominated by photons that are radiated in the low- $\beta$ quadrupoles (where the beam dimensions are large), and reach the IP chamber with grazing angles of a few milliradians after being back scattered from downstream impact points. It has therefore been proposed to shadow the IP beampipe from grazing incidence photons with local absorber rings, made of tungsten [23]. These synchrotron radiation masks should be installed close to the interaction points, inside the experimental vacuum chamber. As they become strong photon scattering sources themselves, additional shielding rings are required outside the vacuum chamber. The expected reduction of the photon background rate with synchrotron radiation masks is about two orders of magnitude. IP masking has been successfully introduced in other electron-positron colliders, for example in the TOPAZ detector at TRISTAN [24], and in the Cornell collider [25].

The longitudinal position and the inner radius of the mask are constrained by several conditions (Fig. 9). In order to obstruct the acceptance of forward physics detectors as little as possible, the thin part of the vacuum chamber should not be reduced to below $\pm 1.8 \mathrm{~m}$, corresponding to a smallest acceptance angle of $30 \mathrm{mrad}$. The inner mask radius must be small enough to cast a shadow for the dominating 2 to $3 \mathrm{mrad}$ incident photons over the complete length of the transparent IP chamber, but must stay outside the very intense direct photon 


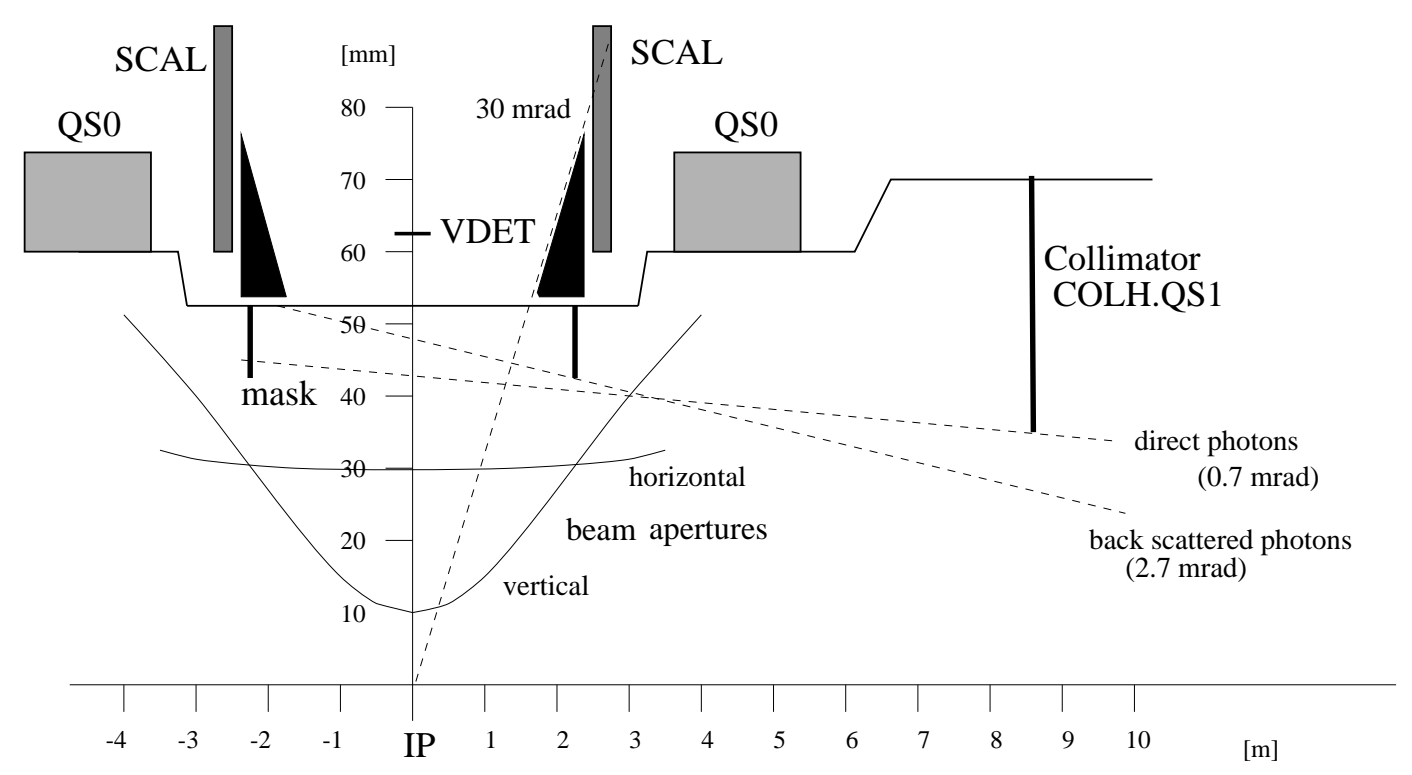

Figure 9: Schematic layout of synchrotron radiation masks around a LEP IP, indicating the boundary conditions for a mask solution: (i) to stay outside the required LEP aperture (solid lines, marked horizontal and vertical), (ii) to cast a shadow over the entire unshielded IP beampipe length for small angle backscattered photons (dashed line), (iii) to stay outside the very intense beam of direct photons collimated by the near $(8.5 \mathrm{~m})$ synchrotron radiation collimator, when closed to 12 beam standard deviations (dashed line).

beam that passes through the minimum opening of $12 \sigma_{x}$ of the near collimator COLH.QS1 at $8.5 \mathrm{~m}$ from the IP. Furthermore, and most important, the mask must stay clear of the required machine acceptance. A geometrical solution, that fulfills all conditions, is shown in Fig. 9. In this "nominal" mask solution [26] the inner mask tip is at $2.3 \mathrm{~m}$ from the IP, while the outer shield starts at 1.8 meters. As the probability to backscatter from the masks into the detector region falls very rapidly with increasing distance from the mask, the $50 \mathrm{~cm}$ long shield upstream of the mask will intercept more than $90 \%$ of these photons. The inner mask radius in this solution is restricted to a range between $40 \mathrm{~mm}$ and $45 \mathrm{~mm}$.

The optimum inner radius for the "nominal" SR mask was found by simulating the shadowing effect of the mask and shield using the Monte Carlo program [7]. Photons incident on the mask or shield are either reflected from the tungsten surfaces or absorbed; transmission through the absorber material has been neglected. The number of simulated SR photons that reach the $3.8 \mathrm{~m}$ long unshielded IP beam pipe is shown in Fig. 10 as function of the mask inner radius for a $90 \mathrm{GeV}$ beam with $50 \mathrm{~nm}$ horizontal and $2.5 \mathrm{~nm}$ vertical emittance. The total background rate is the sum of the components: 


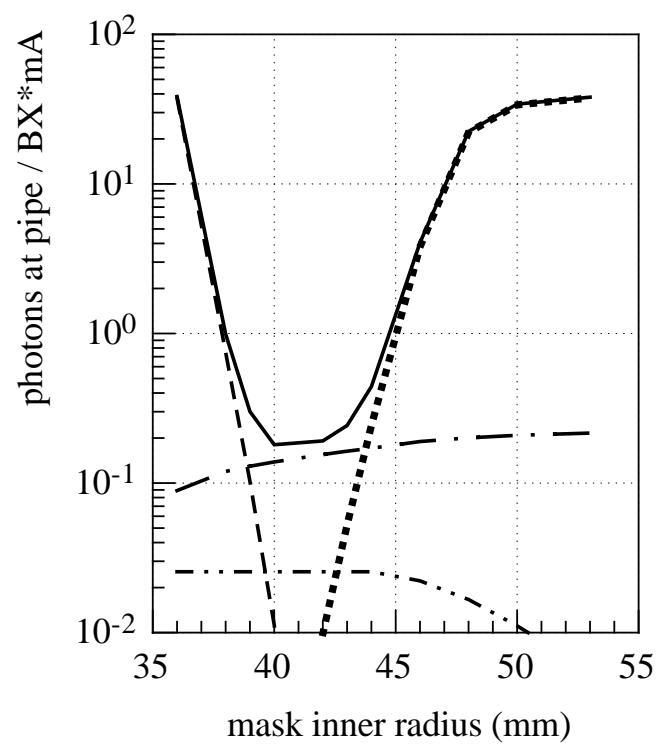

Figure 10: Simulated SR photon background rate incident on the unshielded $3.6 \mathrm{~m}$ long beam pipe as function of the inner radius of SR masks placed at distances of $2.3 \mathrm{~m}$ from the IP. The total photon rate (solid line) is the sum of different components: "small angle" photons backscattered from the QS3 region (dotted), "direct" photons back scattered from the mask and shield (dashed), "large angle" photons scattered from objects closer to the IP (dash-dotted), and "double scattered" photons (dash-double dotted). Conditions: $E_{\text {beam }}=90 \mathrm{GeV}$, $\epsilon_{x}=50 \mathrm{~nm}, \epsilon_{y}=2.5 \mathrm{~nm}$, Gaussian beam density distributions in the horizontal plane, exponential distributions in the vertical plane, COLH.QS1 set at $12 \sigma_{x}$.

- (i) small angle photons arrive with grazing angles of $\approx 2$ mrad after being backscattered from downstream impacts more than $50 \mathrm{~m}$ away. These are the dominant fraction at LEP2 and can be efficiently suppressed with SR masks of $R_{\text {mask }}<43 \mathrm{~mm}$. The small angle photons are radiated mainly from low- $\beta$ quadrupoles on either side of the IP, where the beam dimensions are large.

- (ii) direct photons hit the SR masks and are scattered into the detectors. This background rate rises very sharply and dominates for $R_{\text {mask }}<38 \mathrm{~mm}$. They are radiated from far quadrupoles.

- (iii) large angle photons reach the detectors after being scattered by the near collimators, $8.5 \mathrm{~m}$ from the IP. They arrive with average angles of $10 \mathrm{mrad}$ and can only partly be intercepted by the mask. These photons originate from all quadrupoles along the $250 \mathrm{~m}$ straight section upstream the IP. 
- (iv) double scattered photons arrive at the mask after one scattering on upstream or downstream vacuum elements and a second scattering from the mask into the detectors.

The remaining background is dominated by large angle photons whose flux can be partly controlled by the $8.5 \mathrm{~m}$ collimators. Opening these collimators will reduce the number of large angle photons, but will also reduce the shadow over the mask for direct photons and therefore decrease the width of the minimum in Fig. 10. The shadowing of the mask against direct photons is helped by the new LEP2 collimators COLH.QS3 (Table A.1), and by giving the horizontal jaws of the near collimators a round shape.

The height and width of the minimum in Fig. 10 depend on the longitudinal location of the mask. Moving mask and shield further away from the IP increases the rate at the plateau and reduces the valley width. At the optimum mask radius of $R_{\text {mask }}=42 \mathrm{~mm}$ the theoretical background reduction factor for the "nominal" SR mask is about 200. This optimum figure will be reduced due to several effects, not included in the MC model: (i) non-zero probability of photon transmission through the mask and shield material, (ii) direct photons from quadrupole radiation that can reach the mask edges if large orbit deviations in upstream quadrupoles exist, (iii) multiple scattered photons that arrive with large angles at the IP, (iv) misalignments of the mask and detector pipe with respect to the beam line. The protection strength of the SR mask has been tested in several machine experiments (see section 4.5) and found to be satisfactory [27].

In practice, SR masks and the complementing outer shields are difficult to incorporate into the existing detectors and vacuum layouts. The actual designs of the mask sytems for the four LEP experiments are therefore all different and differ in detail from the ideal nominal design. The main characteristics of the four mask designs are summarised in Table A.3 in Appendix A, where the symbols used are defined in a sketch.

Mask and shield are made of tungsten to maximise their absorption power for low energy photons. The length of the mask (30 radiation lengths) and the thickness of the shield are defined by the required attenuation factor for the high energy tail of the photon spectrum, and by the requirement to absorb shower particles from off-energy beam particles hitting the mask [26]. The central part of the nominal mask is $L=100 \mathrm{~mm}$ long and tilted towards the IP $\left(\gamma=1^{\circ}\right)$ to suppress forward scattering from the mask. Wedge angles of $\alpha=\beta=15^{\circ}$ are chosen to minimise wall effects. Due to installation difficulties the nominal distance $\left(L_{\text {mask }}=2.3 \mathrm{~m}\right)$ of the inner tip of the mask from the IP could not be achieved for the actual installations and several other parameters had to be relaxed (Table A.3), mainly to reduce the weight of the tungsten mask which is supported by the vacuum chamber. The central chamber and the two masks must be aligned parallel to the LEP beams with high precision (to within $0.2 \mathrm{mrad}$ ) in order not to lose the shadowing effect. 
The nominal tapered outer shield along the $30 \mathrm{mrad}$ acceptance line reaches a thickness of $d_{\max }=20 \mathrm{~mm}$. Its shortest distance to the IP $\left(L_{\text {shield }}=1.8 \mathrm{~m}\right)$ is defined by the nominal acceptance angle $\delta=30 \mathrm{mrad}$ and the central vacuum chamber outer diameter of $110 \mathrm{~mm}$. The nominal mask is shadowing the unshielded part of the IP-pipe against photons up to a maximum angle of

$$
\phi_{\text {shadow }}=\left(R-R_{\text {mask }}\right) /\left(L_{\text {mask }}-L_{\text {shield }}\right)=2.66 \mathrm{mrad} .
$$

In practice, a gap of several $\mathrm{mm}$ between chamber and shield is required for installation safety, which reduces thickness and length of the shield. Further reductions in the strength of the outer shield are forced by different layout and installation requirements, in particular in OPAL and L3 (see Table A.3). It has been possible to install the shield with a precision of 0.1 to $0.2 \mathrm{~mm}$ relative to the beam vacuum pipe which holds the mask. The masks have been installed with a precision of about $0.2 \mathrm{~mm}$ relative to the nominal beamline surveyed through the experiments.

A test SR mask in DELPHI was installed in 1994 [23], and masks were available in DELPHI and OPAL during 1995. All four SR masks and shields will be in place for the first LEP2 physics running in 1996.

\section{Backgrounds in the Experimental Detectors}

The main features of the detectors $[28,29,30,31]$ of the LEP experiments will be outlined here with an emphasis on those aspects which are sensitive to backgrounds or which provide information on background conditions. Background monitoring and protection systems within the LEP experiments will be described.

Subdetectors have different sensitivities to background conditions depending on their mode of operation, their role in the experiment, and their location on the beam line. The central detectors of a typical LEP experiment are shown in Fig. 11.

The detectors which are especially vulnerable to background include

- Silicon detectors used as central tracking detectors.

- Gas ionization detectors used for tracking (including drift chambers and Time Projection Chambers (TPC)

- Luminosity calorimeters (silicon, ionization, or scintillation detectors).

Table 4 summarizes the sensitivity of these classes of detectors to the background particles. The most important limit on photon background comes from the operation of gas tracking detectors, while the electron background causes a high spurious trigger rate and degradation of the measurement of luminosity. Although some detectors can respond to photons in the few $\mathrm{keV}$ energy range, photons of such a low energy are very unlikely to penetrate the beam pipe and the support structure of the detectors. Only photons impinging on the beam pipe 


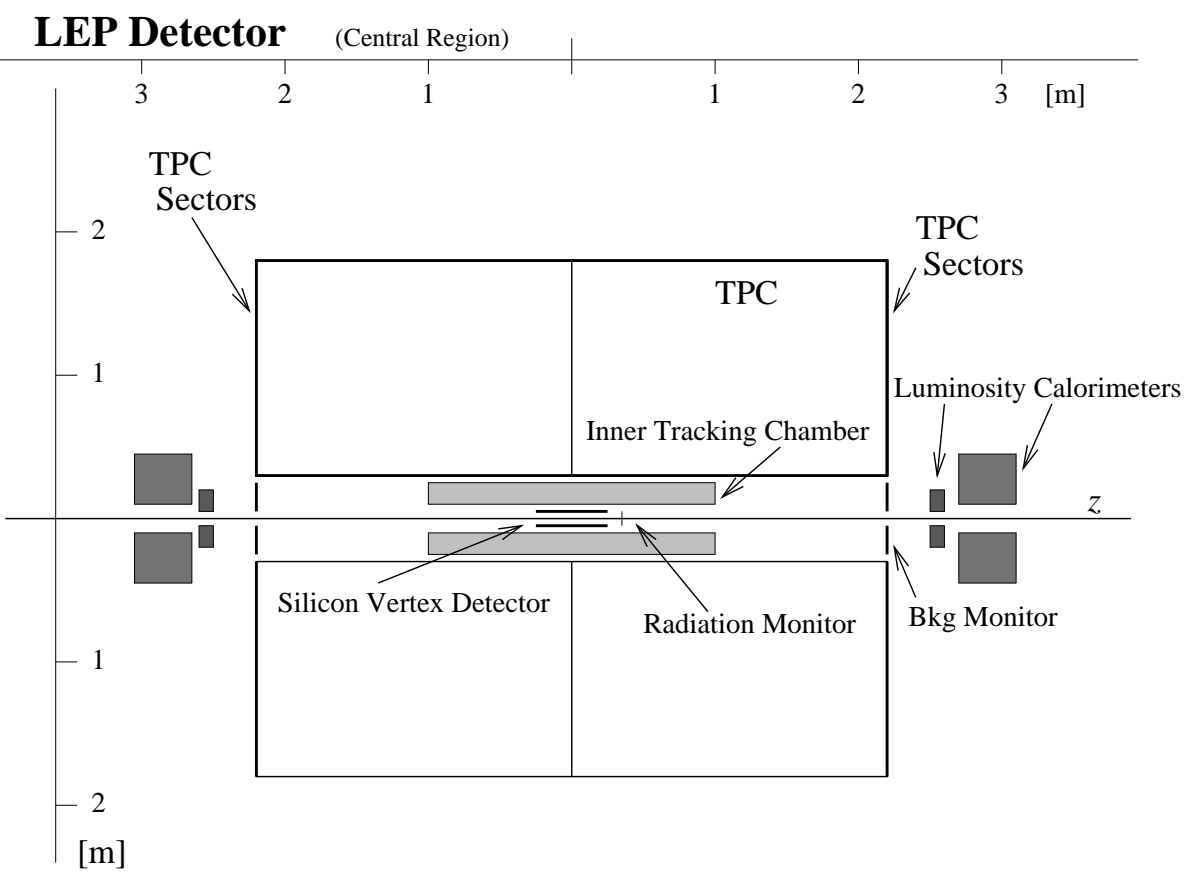

Figure 11: The central subdetectors of a typical LEP experiment showing also the background and radiation monitors. The TPC sectors (wire chambers) are located at both ends of the TPC. Luminosity calorimeters may be based on gas ionization but are often silicon/tungsten devices.

Table 4: Sensitivity of Subdetectors to Background Radiation

\begin{tabular}{|l|c|c|c|}
\hline & Particle type & Minimum Energy & Vulnerability \\
\hline \hline Central Silicon Detectors & photons & $100 \mathrm{keV}$ & Radiation damage \\
\hline Gas Ionization Detectors & photons & $1 \mathrm{keV}$ & Wire damage \\
\hline Luminosity Calorimeters & electrons & $20 \mathrm{GeV}$ & $\begin{array}{c}\text { Luminosity precision, } \\
\text { Spurious triggers }\end{array}$ \\
\hline
\end{tabular}

with energies above $10 \mathrm{keV}$ have a significant probability of being detected. The limits of background which are tolerable for each detector are discussed in the following sections.

The local cartesian coordinate system used to describe the detectors is:

- $z$ lies along the beam direction increasing in the direction of the positron momentum.

- $y$ points upward, perpendicular to the plane of the LEP ring.

- $x$ is radial in the plane of the LEP ring and increases in the outward direction. 
In the local coordinates $\theta$ is the polar angle relative to the positive $z$ direction and $\phi$ is the azimuthal angle measured from the positive $x$ axis.

Tables summarizing the properties of the detectors are given in Appendix B.

\subsection{Vacuum Chamber}

The beam vacuum chamber in the vicinity of the interaction point is made of $1.1 \mathrm{~mm}$ beryllium and has an inner radius of $53 \mathrm{~mm}$. The beryllium section extends to about $400 \mathrm{~mm}$ on each side of the IP and there joins a $1.5 \mathrm{~mm}$ thick aluminum pipe.

Synchrotron radiation photons arriving at the beam pipe at shallow angles have a significant probability of undergoing Compton scattering in the beryllium, with the secondary photons then entering the central region of the detector. Once they reach the higher $Z$ materials beyond the beam pipe the photoelectric effect dominates and the photons are either absorbed in passive material or detected.

\subsection{Silicon Vertex Detectors}

Silicon central tracking detectors are placed as close as possible to the beam pipe to optimize the spatial resolution for identifying secondary vertices due to the decay of short-lived particles. The vertex detector is of key importance for identifying short-lived particles such as the $\tau$ lepton and particles containing $\mathrm{b}$ quarks. It plays a particularly important role at LEP2 as B particles will be an important signature for the appearance of the Higgs [32].

At LEP, several layers are normally installed at radii between $60 \mathrm{~mm}$ and $120 \mathrm{~mm}$ just outside the beam vacuum pipe. The detectors are constructed out of several hundred, $60 \mathrm{~mm}$ square, $300 \mu \mathrm{m}$ thick silicon wafers arranged in "ladders" of four to six wafers around the vacuum pipe.

A bias voltage is applied across the silicon junction to form a depletion region which is sensitive to ionizing tracks. Some of the amplifying and multiplexing electronics circuits are situated at the ends of the ladders. The electronics components, when powered, are vulnerable to radiation damage as are the wafers when the bias voltage is applied.

It is believed that with voltage on, electronics components may be damaged after an accumulated dose of $10 \mathrm{krad}$, while with voltage off the components may withstand $100 \mathrm{krad}$ [33]. The silicon detector wafers themselves are likely to be damaged only by much higher doses. However, incidents have been observed in which very localized damage to electronics components due to sudden beam losses has taken place. In these cases the total dose level is much lower than that expected to cause damage. A decoupling capacitor chip commonly used in such detectors can undergo breakdown due to a sudden large charge deposition such as results from a partial beam loss. 
It appears that during normal operations the radiation load on the silicon detectors is acceptable but that vigilance is required. During the life of the experiments at LEP it is not likely that the accumulated radiation dose during normal operations will cause damage. The risk from sudden beam losses is, however, quite serious and measures (described in section 3.5.6) have been taken to protect against those.

During normal operation the increase in the number of detector hits due to background photons does not compromise the pattern recognition ability.

\subsection{Gas Ionization Detectors}

The drift chambers and the Time Projection Chambers (TPC) are gas detectors filled with argon gas and a small admixture of other gases. Although most such detectors operate at atmospheric pressure, the OPAL CV drift chamber runs at 4 bar. These detectors are designed to be sensitive to energy depositions $\lesssim 1 \mathrm{keV}$ for minimum-ionizing charged particles, and are therefore sensitive to keV photons which interact in the gas or in wires and other local material.

Gas proportional detectors, drift chambers, time projection chambers, and time expansion chambers share common design features. They are provided with very fine (typically $20 \mu \mathrm{m}$ ) sense wires, run at a high positive potential and negative cathode wires or pads. There may be, in addition, field shaping and gating grid wires which are usually of larger diameter and are normally not subject to damage.

Ionization in the gas is created along a charged particle track (from an incident charged particle or from a photoelectron ejected in a photon interaction). The electrons drift in an electric field toward the sense wire. In the immediate vicinity of the sense wire the electric fields are very large and secondary electrons are produced in collisions in the gas. The electron avalanche moves toward the sense wire while the positive charges move in the opposite direction. Electrical pulses are induced on the sense wire and on the cathode wires or pads. These pulses become the signals giving position information and energy information about the primary particle.

A large photon flux causes large currents to be drawn by the sense wires and may result in formation of impurity deposits on the wires or even breakage. An estimated safe limit [34] for wire detectors is an accumulated charge of 0.001 coulombs per mm of sense wire.

In LEP experiments the drift chambers are used for precision measurements of charged particle tracks and are located close to the beam vacuum pipe but beyond the smaller and more precise silicon vertex detectors. 


\subsubsection{The Time Projection Chamber}

At LEP both ALEPH and DELPHI use the Time Projection Chamber (TPC) as an important part of the tracking system. The two TPC's are quite similar but the dimensions for the slightly larger ALEPH TPC will be given here. The ALEPH TPC consists of a gas volume (argon-methane) extending in radius from $0.31 \mathrm{~m}$ to $1.8 \mathrm{~m}$ and $4.4 \mathrm{~m}$ in length. The gas volume is approximately $43 \mathrm{~m}^{3}$. The ionization created in the gas by charged particles drifts in a longitudinal electric field $(11 \mathrm{kV} / \mathrm{m})$ to either end of the device where 18 wire chambers or sectors are situated.

The sector sense wires which detect the ionization clusters are run at a nominal voltage of 1250 Volts providing a gas gain of about $6 \times 10^{3}$. Electrical signals are taken both from the sense wires and from small cathode pads adjacent to the wires. The pads provide hit information for tracking in a simple $r \phi$ coordinate system while the wires provide energy loss or $d E / d x$ information used for particle identification.

The $z$ position of a hit is obtained from the drift time of the ionization cluster in the longitudinal electric field. The drift velocity is about $50 \mathrm{~mm} / \mu \mathrm{s}$ so that the drift time in the TPC is about $44 \mu \mathrm{s}$. With four-bunch operation $(\approx 22 \mu \mathrm{s}$ between beam-crossings) random hits from two beam-crossings are visible in the detector.

\subsubsection{Wire Detector Aging}

Both the sense wires and the cathode wires may become coated with thin layers of substances which are present in the gas or in the construction materials of the detector. If an insulating layer is deposited on the cathode then positive ions reaching the cathode may remain on that layer. The positive ions effectively reduce the work function of the metal and pull electrons out of the surface. These electrons drift to the anode, the process is repeated, and a buildup of current develops.

If an insulating coating develops on the anode wires then the surface electric field is modified by collected electrons and the gain will change, become nonuniform in space, or rate dependent. The measurements of $d E / d x$ depend on stability of gain, and if a conducting coating develops then the effective wire diameter changes locally and the gain may again become non-uniform degrading the $d E / d x$ resolution.

The rate of aging seems to depend on the presence of certain impurities and may vary with gas quality, rate of flow, and materials present. The aging of a chamber depends on the total accumulated charge per unit length of wire; a safe limit appears to be 0.001 coulombs per mm of wire. A year of TPC operation at a very high current $\left(100 \mathrm{nA} /\right.$ sector) is equivalent to about $10^{-5}$ coulombs $/ \mathrm{mm}$. In ten years of such operation one-tenth of the safe limit of 0.001 coulombs $/ \mathrm{mm}$ 
would be accumulated for each sector and failures would be likely.

A gating grid operated synchronously with the beam crossing signal prevents positive ions from returning to the active volume of the TPC; consequently space charge build-up and field distortions are not a serious problem. To reduce the space charge further, the gating grid could be pulsed only on event triggers, not a very desirable mode of operation as it introduces electrical noise.

The number of random hits per beam crossing for any level of current that can be tolerated does not contribute to problems with hit occupancy or pattern recognition in analyzing data. Each charged track that traverses the radius of the detector is defined by about 20 pad hit clusters, which are highly correlated and have very good spatial resolution so that a small number of random hits has little effect.

\subsection{Luminosity Calorimeters}

The Luminosity Calorimeters lie just outside the beam pipe at a distance from the IP of about $2.5 \mathrm{~m}$. The active region extends radially from about $60 \mathrm{~mm}$ to $146 \mathrm{~mm}$ and covers an angular range $(24.3 \mathrm{mrad} \leq \theta \leq 57.7 \mathrm{mrad})$. The calorimeters are designed to detect pairs of Bhabha electrons with very high spatial precision and good energy resolution.

As the Bhabha cross section falls steeply with angle, a precise measurement of luminosity requires both sensitivity at small angles and a very precise determination of the inner edge of the detector acceptance. The detector acceptance $A$, (the cross section for Bhabha events which are observed) is given by $A[\mathrm{nb}]=1040\left(\theta_{\min }^{2}-\theta_{\max }^{2}\right) / s$ where $s$ is the square of the center-of-mass energy $[\mathrm{GeV}]$. Angular cuts made in software restrict the inner angle to $\theta>30 \mathrm{mrad}$ with very good precision although some experiments use a precise massive shield to define the edge.

Since the Bhabha electrons are produced at the beam energy, good energy resolution helps to suppress background. Radiative processes, however, result in a low energy tail on the Bhabha events, blurring the separation between those events and off-energy electrons. Off-energy electrons lead to a high trigger rate and also can be superimposed on single-arm Bhabha events.

Luminosity calorimeters are also sometimes placed at 7 to 8 meters from the interaction point beyond the low- $\beta$ quadrupoles where they accept smaller angle Bhabha electrons at a high rate but are also subject to higher off-energy background. (See Fig. 7 and Section 2.4.1.)

\subsection{Background Monitoring}

In each of the LEP experiments two background figures-of-merit are computed based on its own subdetectors. One figure-of-merit ("bkg1") represents the rate of low energy photons while the second figure-of-merit ("bkg2") represents the 
rate of off-energy electrons. The figures-of-merit are normalized to a scale in which "1" means that conditions are good and "5" means that the background is too high for normal operation. The values are not normalized to beam current as they are intended to represent the level of risk to the detectors.

The values are transmitted to the LEP data base system and are displayed in the LEP control room. These indicators are used by the LEP operators to optimize the background conditions and by the experiments' operations crew to decide when to raise the high voltage of sensitive detectors at the start of physics data-taking. Additional monitoring systems provide estimates of instantaneous and accumulated radiation doses to the silicon detectors.

\subsubsection{Photon Rate Monitor}

The principal operational constraint to bringing the experimental detectors up to operating conditons at the start of a fill is the current drawn by drift chamber sense wires. High current is almost always an indication of a large soft photon flux therefore the photon monitor signal "bkg1" is derived from either the sense wire current or from the random hit rates in wire detectors.

\subsubsection{Electron Rate Monitor}

The electron rate signal "bkg2" is derived from luminosity calorimeters which are sensitive to off-energy electrons above about $20 \mathrm{GeV}$. The significance of the offenergy electron background depends on the nature of the luminosity triggers and on algorithms used to deduce the luminosity. During periods of poor vacuum conditions in the distant part of the straight-section, single electron rates needed to interpret the luminosity have become unacceptably high. The luminosity detectors provide an accurate indication of the off-energy electron contamination and can give detailed spatial and energy distributions of the electrons for comparison with simulations.

\subsubsection{Multi-Purpose BaCkground Monitors}

A very robust thin drift chamber which is sensitive to electrons as well as to photons provides an indication of background under conditions too severe for other detectors to be brought up to normal voltage. This is often the case just after injection and during acceleration of the beams (the start of a "fill") and this signal can be used to assist in adjusting beams in LEP.

As this detector is very thin it has a low efficiency for detecting photons but is fully sensitive to electrons. The photon detection efficiency is enhanced by a thin layer of copper (on the cathode pads) in which primary photons interact via the photoelectric effect. The emerging copper x-ray (about $8 \mathrm{keV}$ ) has a higher probablility $\left(\approx 10^{-3}\right)$ to be detected in the gas volume. 
The detector is effective for studying the time-of-arrival of photons and has been important for identifying backscattered synchrotron radiation from distances up to $100 \mathrm{~m}$.

A new monitor with double planes has been installed in ALEPH in 1996 to permit event-by-event discrimination between photons and electrons. It has improved time resolution to distinguish among families of backscattered photons.

\subsubsection{Photon Arrival Time}

Gas ionization detectors are able to measure the time of arrival of photons relative to the crossing time of the electron-positron beams at the IP. Photons which arrive directly from their source in magnetic fields or which are forward scattered arrive in time with the beam crossing ("prompt photons"). Photons which are back scattered from collimators or other beam-line elements arrive with a delay equal to twice the transit time to the scattering element. The closest object (aside from the masks) which can backscatter is the collimator at $8.5 \mathrm{~m}$ from the IP, and these photons, delayed by about $50 \mathrm{~ns}$, can be identified in some subdetectors. Photons backscattered from over 100 meters away have been observed.

For drift velocities of about $5 \mathrm{~cm} / \mu \mathrm{s}$ in a drift cell of about $1 \mathrm{~cm}$ (characteristic of the ALEPH ITC and the small angle background monitor), the drift times extend to about $200 \mathrm{~ns}$ but the drift time distribution from a point source of photons has a sharp leading edge which makes it possible to identify sources with good spatial resolution. Time distributions obtained with the OPAL detector are shown in Fig. 12.

\subsubsection{RADiATiOn MONitor}

Silicon PIN photodiodes used as radiation monitors are located at about the same radius as the vertex detector (see Fig. 11). Four diodes are used for monitoring accumulated radiation doses with a "slow" electronics system. These diodes are $1 \mathrm{~cm}^{2}$ in area and are $170 \mu \mathrm{m}$ thick. They are located about $36 \mathrm{~cm}$ from the IP along the momentum direction of electrons at a radius of about $8 \mathrm{~cm}$. The diodes have been calibrated with radioactive sources and their principal function is to estimate the total dose delivered to the silicon vertex detector. As the monitor diodes are made of the same material as the vertex detector, the estimate of delivered dose to the silicon vertex detector is reliable and does not require detailed information about the photon spectrum. This system is continually active and the instantaneous dose as well as the history of the dose labeled by the LEP operating mode are always available.

\subsubsection{BEAM DUMP}

Beam dump systems in the LEP experiments were commissioned in late 1992 and 1993 and have been in use since then. They detect a sudden increase in 


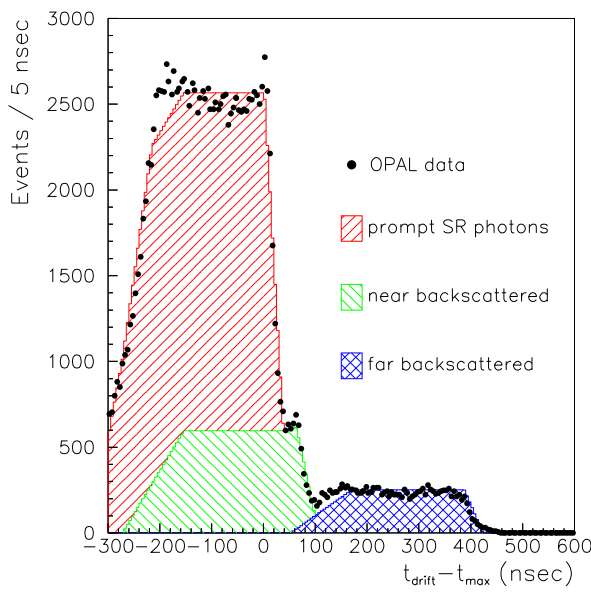

Figure 12: Distribution of arrival times (relative to the maximum drift time for the hit wire) of synchrotron photons in the OPAL vertex drift chamber. The data points are data from the 1992 run. The different cross-hatched areas represent the contributions from prompt and near- and far-backscattered photons. The time delay for near-backscattered photons was $60 \mathrm{ns,} \mathrm{corresponding} \mathrm{to} \mathrm{backscattering}$ from a distance of about $9 \mathrm{~m}$. For the far-backscattered photons, the time delay was $385 \mathrm{~ns}$, corresponding to about $60 \mathrm{~m}$.

radiation close to the beam pipe and trigger a dump of the LEP beams within a few milliseconds. Once the radiation level detectors had been made fail-safe with redundant electronics, no spurious dumps were initiated and it is estimated that the radiation dose to the detectors was reduced by at least a factor of two. About 10 physics fills per year are dumped by the LEP experiments

PIN photodiodes mounted close to the interaction point form the detection end of the beam dump system in ALEPH. Their charge is integrated by preamplifiers with a characteristic integration time of $100 \mu$ s (approximately one turn of LEP) and are sensitive only to high doses received in a short time interval. The dumping of the beams was originally implemented by switching off RF and more recently by triggering the beam kicker system.

The threshold to initiate a dump corresponds to 0.75 mrads per $100 \mu \mathrm{s}$ or 7.5 rads per second received within $100 \mu \mathrm{s}$. The beam dump system is not activated unless both the vertex detector high voltage and the vertex detector electronics low voltage are on.

In OPAL the silicon tungsten luminosity calorimeter has been used to trigger beam dumps, requiring a coincidence between large bias currents in odd and even layers. A dump was triggered when the equivalent of $10^{7} 45 \mathrm{GeV}$ electons was detected in one second. A link to the fast beam kicker dumped the beam in $400 \mu \mathrm{s}$. This system was active at all times. For LEP2 running this dump system 
is being replaced by a set of silicon radiation monitors. A dump will be triggered on a dose rate of $10 \mathrm{rad} / \mathrm{s}$ in one monitor and at least $7.5 \mathrm{rad} / \mathrm{s}$ in a second one.

In the L3 experiment silicon diodes at a radius of $85 \mathrm{~mm}$ are used as the radiation monitor [35]. The low threshold beam dump trigger is set for $6 \mathrm{rad} / \mathrm{s}$

for at least $300 \mu \mathrm{s}$ on two out of four channels. This was active when sensitive detector high voltages were on. A dump threshold 100 times higher was active at all times.

\subsection{Background Simulations in the Experimental Detectors}

The detailed simulation of the photon background in LEP (see Sect. 2.2.2) provides photon energy spectra and rates for photons hitting the vacuum pipe near each experiment. Most background photons can be classified into four families depending on their origin and angular distribution:

- forward scattering from collimator at $8.5 \mathrm{~m},<\theta>=10 \mathrm{mrad}$.

- backscattering from collimator at $8.5 \mathrm{~m},<\theta>=10 \mathrm{mrad}$.

- backscattering from $55 \mathrm{~m}$ region, $<\theta>=2 \mathrm{mrad}$.

- direct photons from distant quadrupoles, $\langle\theta>=0.3 \mathrm{mrad}$.

The Monte Carlo simulations of the experimental detectors use as input the photons hitting the beampipe within $\pm 2.4 \mathrm{~m}$ of the IP. These incoming photons are given the energy distribution and the incident angles characteristic of each of the families. In an alternative approach the incident photons are given the characteristic angles but are assigned discrete energies over the entire range of interest. Fig. 13 gives the detection probability in ALEPH as a function of energy for photons which hit the beam pipe. These have been obtained using the ALEPH detector simulation code which is based on GEANT [36]. The detection probability and distributions for each energy are then folded with the spectra for each of the families.

The photons are tracked through the beam pipe and the detector using detailed simulations of the detector material and geometry. The energy deposited in the sensitive volumes of the detector elements is tabulated, and detector "hits" are generated if the particular detector should have been sensitive to the deposited energy. It is necessary to track particles and to record energy depositions as low as $1 \mathrm{keV}$, since the gas ionization detectors are sensitive to energy losses of that magnitude.

Hit rates and position distributions of photon hits are available for the central detectors for each family of photons. The simulated hit rates are folded with the detection probabilities for the central detectors obtained from a detailed detector simulation code. These photon detection efficiencies for several families of photons are given in Table 5 for the central detectors of DELPHI [37] and OPAL [38]. 


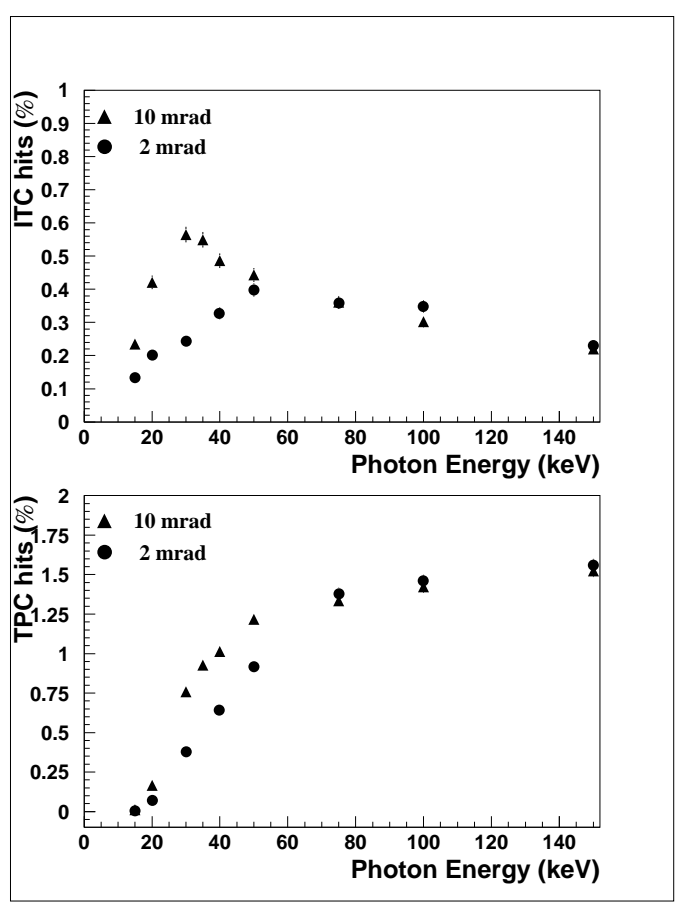

Figure 13: Percentage of those photons hitting the beam pipe which are recorded in the ALEPH ITC and TPC as a function of photon energy for incident angles of $2 \mathrm{mrad}$ and $10 \mathrm{mrad}$.

Table 5: Photon Detection Efficiency.

\begin{tabular}{|l|c|c|c|c|}
\hline Photon family & DELPHI TPC & DELPHI VD & OPAL CV & OPAL CJ \\
\hline $\begin{array}{l}\text { Forward scattered photons } \\
\text { hitting beam pipe }\end{array}$ & $1.910^{-2}$ & $0.610^{-2}$ & $0.8110^{-2}$ & $7.110^{-2}$ \\
\hline $\begin{array}{l}\text { Backward scattered photons } \\
\text { hitting beam pipe }\end{array}$ & $3.610^{-2}$ & $1.410^{-2}$ & $1.110^{-2}$ & $3.310^{-2}$ \\
\hline Direct photons hitting mask & $1.010^{-5}$ & $1.110^{-6}$ & $2.610^{-7}$ & $2.110^{-6}$ \\
\hline
\end{tabular}

\subsubsection{Estimate of Photon Rates}

The ALEPH TPC has a gas gain of about $6 \times 10^{3}$. A wire current of $100 \mathrm{nA}$ corresponds to approximately $10^{8}$ primary charges. In argon gas $26 \mathrm{eV}$ are required to produce an ion pair so this number of primary charges can be produced by about 50,000 interacting photons per second with an average energy of $50 \mathrm{keV}$. At the typical beam crossing rates at LEP this corresponds to one interacting photon per beam crossing or about 100 photons incident on the beam pipe. 


\section{Background Measurements}

\subsection{Aperture Limits}

There are unavoidable losses of beam particles in scattering processes where the energy loss exceeds the acceptance of the radio frequency acceleration system (typically $1 \%$ of the beam energy). Particles with energy losses of more than $3 \%$ of the beam energy will be lost in the LEP arcs. Particles with energy loss in the range of $1 \%$ to $3 \%$ of the beam energy can make several turns in LEP. These particles can be considered as contributing to the non-gaussian tails in the energy distribution. On a lower level, but well measurable with loss monitors, there are also non-gaussian horizontal and vertical tails, even if beams are not colliding. The far transverse tails are produced by the combined effect of energy loss and dispersion (mainly horizontal energy dispersion in the arcs of LEP).

Aperture collimators serve to remove particles that would be lost anyway; and to provide the required shadow for background collimators to approach the beam without scraping beam tails. They are located sufficiently far from the experiments that they do not produce background. The aperture collimators are equipped with beam-loss monitors. Background rates in the experiments and in the LEP luminosity monitors as well as beam-loss rates at the aperture collimators have been measured for various collimator settings [39].

The main conclusions are:

- The main role of aperture collimators is to protect experiments against background spikes. Under stable running conditions, the use of aperture limiting collimators leads to only a minor reduction in the background as observed in the experiments.

- The off-energy aperture collimators at the end of the arcs (see Table A.1) on either side of the experiments reduce very substantially the background recorded in the LEP luminosity monitors.

\subsection{Off-energy background}

\subsubsection{Origin And CONSEQuences}

The off-energy backgrounds observed by the LEP experiments originate from bremsstrahlung energy loss of beam electrons or positrons, due to interaction with residual gas molecules in the arcs and straight sections upstream of each experiment. Bunch train separation bumps tend to increase the off-energy background rate and shift part of it from the horizontal into the vertical plane [40].

Such backgrounds affect the small angle luminosity monitors, where they can

contaminate the pure sample of Bhabha scattering events needed for luminosity measurement, and produce backgrounds to forward neutral energy triggers. Offenergy electrons are also seen at a low rate in the tracking chambers, where 
they cause high hit multiplicities and therefore contribute to overall ionisation load and misreconstruction of the tracks of charged particles originating from the interaction point.

\subsubsection{Summary OF OBSERVATIONS}

Despite different optics all experiments observe the same general features of offenergy background in their low angle luminosity monitors and tracking chambers. However, the detailed features of the angular and energy distribution of the electrons observed in the luminosity monitors vary significantly from fill to fill. High off-energy rates have been observed during the first weeks of running, after a long shutdown in experiments where large parts of the surrounding vacuum system had been opened to air. The increase in the off-energy rate is due to the larger dynamic vacuum pressure due to components with less cleaned vacuum elements, and shows the typical quadratic dependence on the beam current.

The observed background in the luminometers is uniform in azimuth with low intensity horizontal and vertical bands superimposed and a broad energy spectrum up to $30 \mathrm{GeV}$. The backgrounds in tracking chambers show a similar angular structure. Measurements and simulations of off-energy backgrounds due to bunch train separation bumps are described in section 4.4.3.

\subsubsection{EFFECT OF OFF-ENERGY ELECTRONS ON LUMINOSITY MEASUREMENTS}

The DELPHI VSAT system is made of four $\mathrm{Si} / \mathrm{W}$ calorimeters located beyond the QS0 quadrupole. The luminosity measurement relies on the subtraction of accidental Bhabha concidences. There is a special trigger which allows the recording of "fake" Bhabha events in order to subtract their contribution to the Bhabha distributions. In normal conditions, the fake trigger rate is less than $0.5 \%$ of the Bhabha trigger. After the luminosity selection about $0.1 \%$ of selected events are found to come from accidental coincidences.

In the first fills of 1994, however, because of bad dynamic vacuum conditions, their contribution increased up to $45 \%$ of Bhabha triggers and to $30 \%$ of selected Bhabha events. Fig. 14 shows the "normal" Bhabha correlations, while Fig. 15 shows the background from accidental coincidences. Simulation of the trigger performance indicates that no luminosity measurement useful for high precision

cross-section evaluation is possible with a contribution of fake coincidences to the Bhabha trigger rate of more than 5\% [42].

\subsection{Arc Dipole Photons}

Arc dipole photons originate in the last strong bend dipole at the end of the arc. A very unlikely double scattering would be required for them to reach the beam 


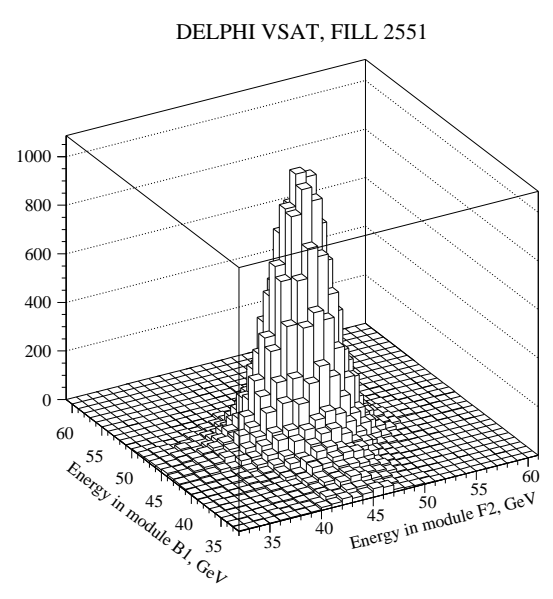

Figure 14: Energy-energy correlation in Bhabha events for "normal" running conditions.

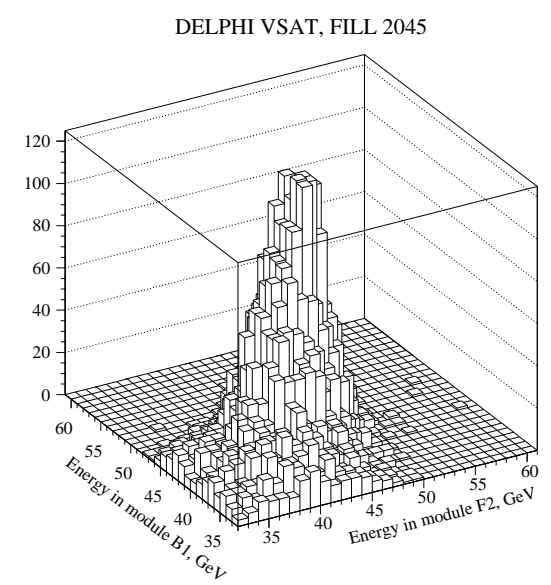

Figure 15: Energy-energy correlation in Bhabha events in conditions of significant off-energy background.

pipe in the vicinity of the IP. However for certain ranges of photon energies they may undergo a mirror or specular reflection on the vacuum chamber elements at about $200 \mathrm{~m}$ from the IP, and reach the region near the IP with a very much enhanced probability. Photons impinging on a surface with an angle below the "critical" angle are reflected with nearly $100 \%$ probability. The critical angle has a simple dependence on photon energy [43] given by $\theta_{c}[\mathrm{mrad}]=33 / E_{\gamma}[\mathrm{keV}]$. 


\subsubsection{Evidence For Arc Dipole Photons}

Measurements of synchrotron radiation background in ALEPH prior to 1993 had revealed two puzzling observations. The observed rate of synchrotron photons was far in excess of Monte Carlo predictions based on a model of the beam and straight-section and on a simulation of the central detector. Furthermore the rate of prompt photons (in time with the electron and positron beams) was much larger than expected, compared with the rate of delayed backscattered photons [44, 52].

This large prompt component motivated an investigation of photons originating in the last bending magnets in the arcs. Collimators had been installed in the ALEPH straight-section at about 235 meters and it was found that these can intercept a significant photon flux. Fig. 16 shows the ITC current (which is very sensitive to low energy photons) as a function of the horizontal QS11 ${ }^{3}$ collimator position. The sharp drop indicates that photons in a narrow angular range are able to reach the central detector. It is believed that these low energy photons are specularly reflected from distant vacuum pipe, and reach the central region after one more scattering with a much higher probability than is possible through Compton scattering alone. The first reflection takes place at about $200 \mathrm{~m}$ and the second at about $50 \mathrm{~m}$ from the IP (see Fig. 2).

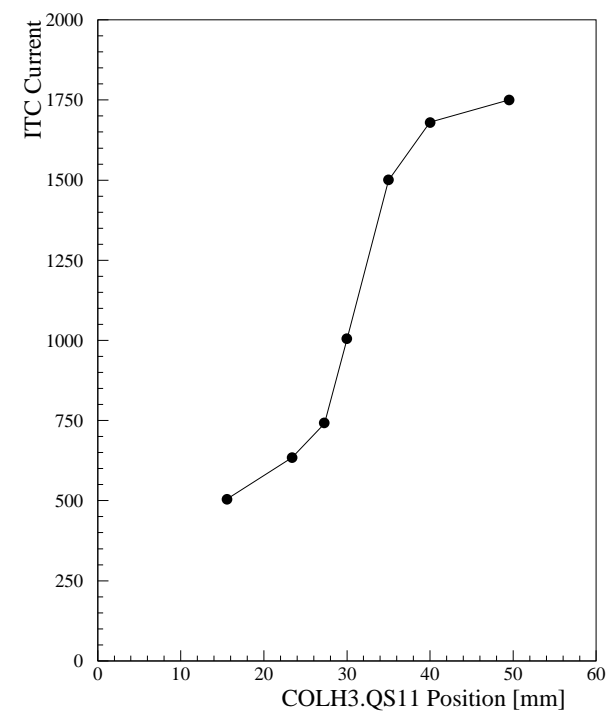

Figure 16: The ALEPH ITC current as a function of opening of collimator, QS11, at $235 \mathrm{~m}$. The sudden drop suggests that a narrow angular range of photons is responsible. In certain regions of the ITC the fractional drop was even larger.

By setting the distant collimator appropriately an immediate reduction in

\footnotetext{
${ }^{3}$ This collimator is a test collimator for electrostatic separators only available on the right side of ALEPH.
} 
background was achieved. Subsequently collimators COLH.QS10 at 220 meters were installed in all the experimental straight-sections.

\subsection{Bunch Trains}

Two schemes with equidistant trains of bunches in each beam have been tried in LEP. In order to avoid additional beam-beam interactions, bunches of counterrotating trains must be separated at their parasitic crossings on each side of the IP's. The separation is produced by electrostatic closed orbit bumps. The first scheme used a $240 \mathrm{~m}$ long separation bump in the horizontal plane, antisymmetric around the IP, which introduced horizontal crossing angles at the IP [2]. The second scheme applied short vertical separation bumps between QS2 and QS7, symmetric around the IP, with zero bump amplitude through the low- $\beta$ quadrupoles QS1 and QS0 and head-on collisions [3]. Due to the presence of the separation bumps additional background at the experiments is generated in both bunch train configurations. More off-energy particles, which are produced along the straight sections, are directed by the bumps into the detectors and many more SR photons are radiated in quadrupoles within the separation bumps, as the beam particles traverse these quadrupoles off-axis where they experience higher magnetic fields. Furthermore, photons from the bump regions are radiated under larger angles with respect to the central beam line, and consequently more photons strike vacuum elements in the vicinity of the IP and are scattered into the experiment. The bunch train configuration with horizontal crossing angles had to be abandoned because of the very large SR photon background rate produced.

\subsubsection{BunCH TRAins With HORIZONTAL CROSSING ANGLES}

In the case of bunch trains with horizontal crossing angle the separation bump spans the entire straight section and reaches amplitudes of more than $10 \mathrm{~mm}$ in the QS1 quadrupoles. Monte Carlo simulations predict for this scheme an increase of the synchrotron radiation photon background by more than two orders of magnitude [45].

The photon background rate with a horizontal angle at the IP was measured during a dedicated machine experiment in 1993 [46]. As the electrostatic separator system was not yet in place, preliminary asymmetric orbit bumps were set up with magnetic corrector elements around the four IP's. This bump, however, was substantially shorter than the bump planned for use which is to be excited with bunch train separators. Therefore the observed background levels are likely to underestimate the rates to be expected with the nominal separation scheme.

Photon rates in all four experiments were measured as function of the horizontal slope $x^{\prime}$ of the orbit at the IP, where $x^{\prime}$ is half the beam crossing angle. A steep increase of the photon background rate with increasing slope is seen in all experiments. The experimental points together with results of Monte Carlo 
simulations [47], modelling the experimental conditions as closely as possible, are given in Fig. 17.

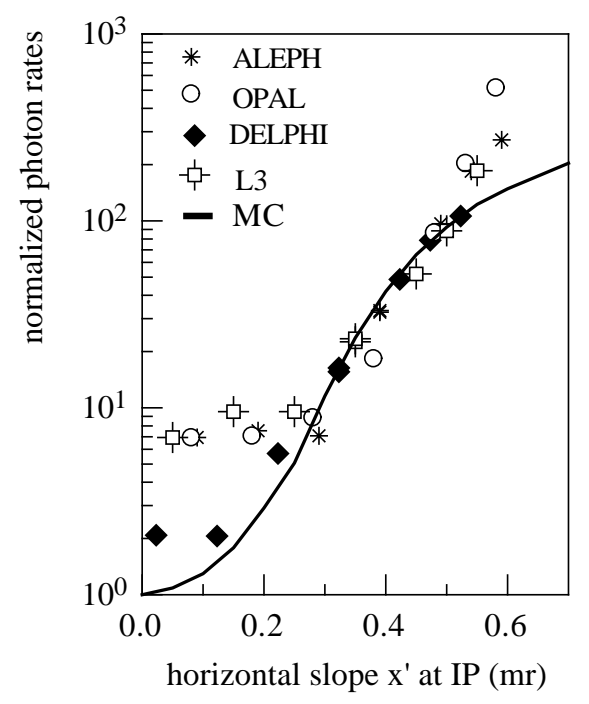

Figure 17: Measured and simulated photon background rates as function of the slope of the asymmetric horizontal orbit bump in the IP's. All rates are normalised (see text).

To compare the figure-of-merit data ("bkg1") from the different detectors with each other and with the MC results, all rates have been scaled to be equal at $x^{\prime}=0.5 \mathrm{mrad}$, corresponding to the nominal $1 \mathrm{mrad}$ crossing angle, and are then normalised to a predicted rate of 1.0 at zero angle. Relative photon rates from the four detectors agree well over the complete angular range, apart from DELPHI, where smaller rates are seen for slopes below $0.2 \mathrm{mrad}$. Good relative agreement between data and MC simulations is obtained for angles between $0.2 \mathrm{mrad}$ and $0.5 \mathrm{mrad}$. For larger slopes the MC modelling of the orbit is no longer sufficient and the predicted rates are too low. For slopes below $0.2 \mathrm{mrad}$ the measured photon rates remain nearly constant. This is due to an additional photon contribution that was not simulated in the model and is independent of the orbit through the straight section. It was found in a later machine experiment that photons radiated from arc dipoles can reach the experiments after a specular reflection plus an incoherent Compton scatter on vacuum chambers (see section 4.3).

The additional photon background from each beam is mainly due to SR in low$\beta$ quadrupoles on both sides of the IP. Therefore, closed orbit manipulations that reduce the offset in quadrupoles for the incoming beams, but keep the crossing angle unchanged, do not help. 
The reasonably good agreement between the relative increase of measured and predicted photon rates under the specific experimental conditions gave confidence in $\mathrm{MC}$ predictions of the photon backgrounds to be expected with crossing angle bunch trains. These calculations predict a photon rate with nominal separator bumps and $1 \mathrm{mrad}$ crossing angles about 400 times the rate obtained with flat orbits. This is far above the level that can be accepted by the LEP detectors and therefore ruled out the bunch train scheme with horizontal crossing angles in LEP [48].

\subsubsection{BUNCH TRAINS WITH HEAD-ON COLLISIONS}

In this scheme the separation of bunches at their parasitic collision points is provided in the vertical plane by much shorter electrostatic orbit bumps, which leave the beams unperturbed in the region of the strong low- $\beta$ quadrupoles QS0 and QS1. One can therfore expect less serious problems from SR background. This was confirmed by several measurements of the photon background with vertical bunch trains. However these measurements revealed a very rapid rise of the detected photon rate with bump amplitudes above a threshold value.

The shape of the vertical separation bump in IP8 is shown in Fig. 18 indicating the locations of the driving electrostatic separators (ES), straight section quadrupoles (QS) and vertical collimators (COLV and COLZ). Symmetric separation bumps of two different amplitudes are drawn with maximum bump amplitudes at QS4 of $11 \mathrm{~mm}$ (solid line) and $9.3 \mathrm{~mm}$ (dotted line). Central directions of some of the photon fans radiated from quadrupole or separator fields by the incoming beam for the two bumps are also indicated (thin full and dotted lines). Photons that hit vacuum elements further than $45 \mathrm{~m}$ from the IP must scatter at least twice to reach the detectors. However, photons that hit elements closer to the IP, in particular, collimators COLV.QS1 or COLZ.QS2, have a high probability to be reflected back into the detectors. One can therefore conclude from Fig. 18 that major additional photon background rates will occur only for bump amplitudes larger than some threshold value, which lies between $9 \mathrm{~mm}$ and $11 \mathrm{~mm}$ in QS4.

The maximum achievable bump at $45.6 \mathrm{GeV}$ with full separator strength (100\% bump) in IP4 and IP8 is $9.3 \mathrm{~mm}$, while in IP2 and IP4, which have different optics, the maximum (100\%) bump reaches $12.6 \mathrm{~mm}$ therefore larger problems from SR photons must be expected in the latter regions if the maximum achievable separation is needed.

The above geometrical explanation of the threshold behaviour of the photon rate was confirmed by MC simulations [45]. For this pupose the MC code [7] was modified to include the effects of electrostatic separators. An example is shown in Fig. 19, giving the total photon rate and its main components as function of the vertical bump amplitude in IP4. The exponential rise of the photon rate for orbit amplitudes larger than $10 \mathrm{~mm}$ is due to back scattered photons from the downstream vertical collimators +COLV.QS1 and +COLZ.QS2. At even larger bump 
amplitudes photons reach the jaws of the upstream collimators -COLV.QS1 and arrive at the detectors with high probability after being Compton scattered in the forward direction. The contribution from the horizontal plane is nearly independent of the bump amplitude and dominates the background rate for amplitudes below the threshold bump amplitude.

Experimental results from a measurement of the photon background rate in ALEPH and DELPHI with vertical bunch trains are reported in Fig. 20, and are compared with MC predictions. The experimental conditions for the experiment

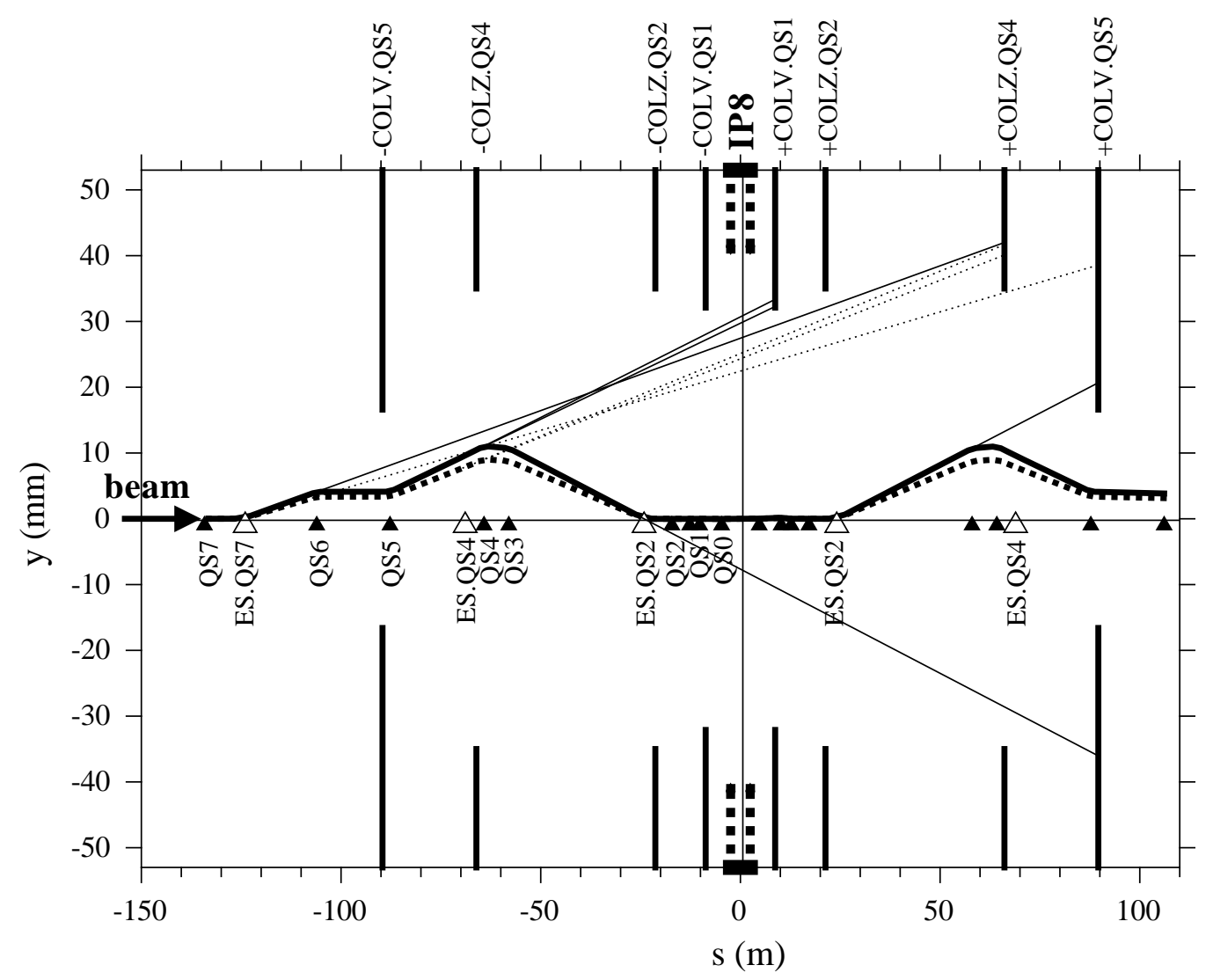

Figure 18: Vertical separation bump around IP8 for the positron beam with 1995 LEP optics. The closed symmetric bumps on either side of the IP are produced by three pairs of separators, ES.QS2, ES.QS4 and ES.QS7. The counter rotating electron beam travels on an orbit, mirror reflected to the central beam line. The bunch separation is therefore twice the orbit amplitude at the location of parasitic collisions. The directions of fans of photons radiated in quadrupoles and separator fields by the incoming beam are given for two bumps with different amplitude (thin full and dotted lines). 


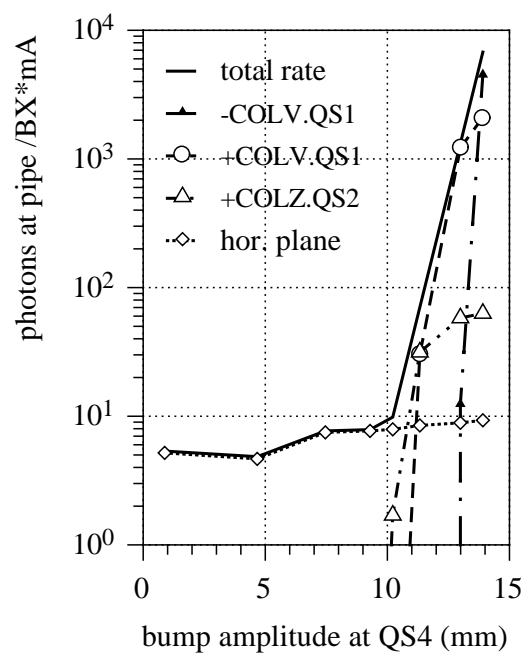

Figure 19: Simulated SR photon rates as function of the separation bump amplitude in IP4. $\left(E_{\text {beam }}=45.6 \mathrm{GeV}, \epsilon_{x}=33 \mathrm{~nm}, \epsilon_{y}=1.5 \mathrm{~nm}\right.$, COLV.QS1 $=35 \mathrm{~mm}$, COLZ.QS2 $=35 \mathrm{~mm}$ ). The total rate is given as well as the contributions from scattering from individual collimators.

are described in detail in [49]. Beams, of one train of four bunches each, were colliding in IP4 and IP8. Bunches were separated at parasitic collision points by the standard electrostatic separator system, and photon rates were recorded as a function of the maximum separation amplitude in QS4. Photon rates plotted in Fig. 20 are normalised, scaled figure-of-merit numbers ("bkg1") from the experiments. Good relative agreement is obtained between measurement and simulation, showing the typical threshold effect.

The actual value of the threshold amplitude depends on the opening of the vertical collimators, mainly COLV.QS1 and is reduced with smaller collimator openings. However, this collimator is required to protect the experiments from additional off-energy background (see below), generated by the separation bump, and therefore must be closed as much as possible. The amount of SR-photon background from the separation bump depends also on the electron beam size and transverse density distribution. With bump amplitudes and collimator openings adjusted to safely stay below the photon threshold, large non-gaussian tails or sudden blow-up of the vertical beam size can shift conditions into the high background region and lead to large spikes in the photon background. This phenomenon has been observed frequently during physics data-taking with bunch trains in 1995.

The very high photon background rate from large beam displacements through 


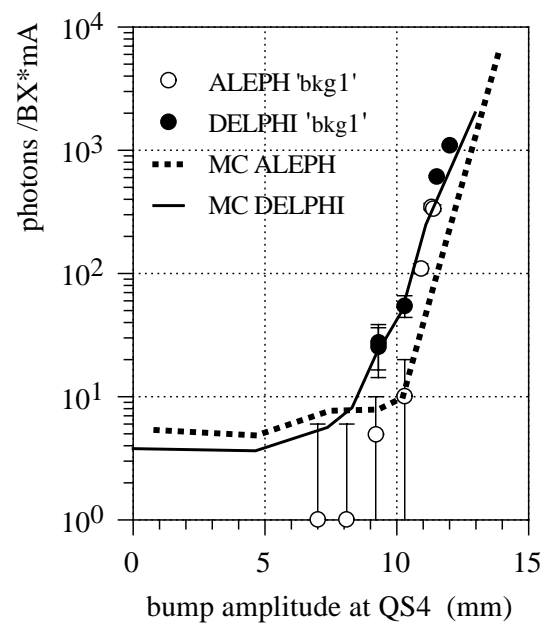

Figure 20: Measured and simulated photon background rates as function of the amplitude of a vertical separation bump in IP4 and IP8. The figure-of-merit numbers "bkg1" are scaled to fit the simulations.

the straight-section quadrupoles can, in principle, be reduced by superimposing antisymmetric magnetic orbit bumps onto the electrostatic separator bumps. The separator bumps are symmetric in the sense that the electron and positron beams have the same displacement amplitude on both sides of the IP. A superimposed antisymmetric magnetic bump allows a reduction of the excursions of the incoming beams on both sides, while keeping the beam separation constant (Fig. 21). The increased radiation generated by the larger excursions of the outgoing beams will produce many more backscattered photons. However, the probability for these photons to reach the detectors is small due to the large distance between the point of impact and the IP, and because more than one scattering is needed to reach the detectors.

The effect of antisymmetric (correction) bumps has been simulated and compared to measurements $[45,50]$ (Fig. 22). Starting with a bump amplitude of $12.6 \mathrm{~mm}$ for the incoming positron beam (100\% bump in IP6), which is well above the threshold amplitude, the photon rate could be lowered by more than an order of magnitude, down to acceptable levels, by superimposing an antisymmetric correction bump of $3 \mathrm{~mm}$ to $4 \mathrm{~mm}$. Again, simulations give good agreement with the experimental results.

The superimposed antisymmetric magnetic bumps, however, produce unwanted vertical dispersion and were therefore avoided during 1995 physics data taking as much as possible. Instead, the beam separation around the IP's was chosen to stay well below the critical threshold bump amplitude of about $9 \mathrm{~mm}$. 

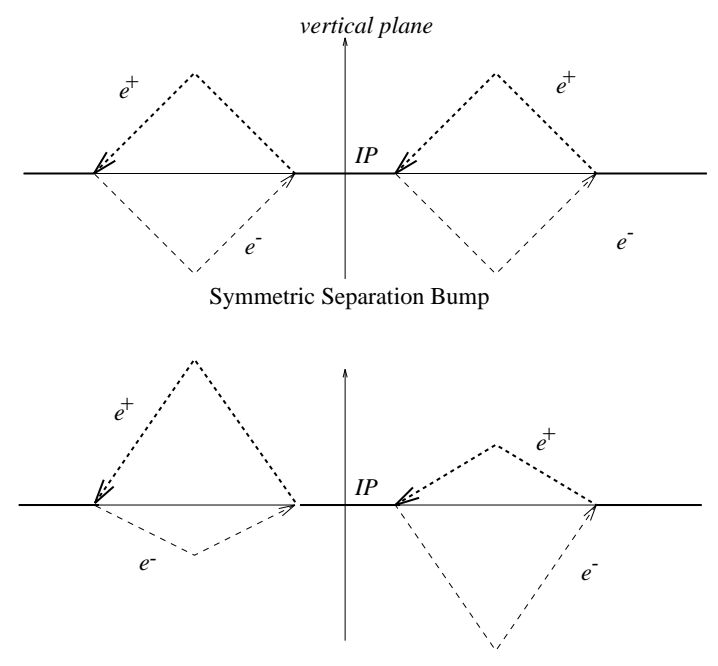

Asymmetric Separation Bump

Figure 21: Schematic presentation of symmetric and antisymmetric vertical separation bumps. In the antisymmetric case the incoming bumps are smaller for a constant separation.

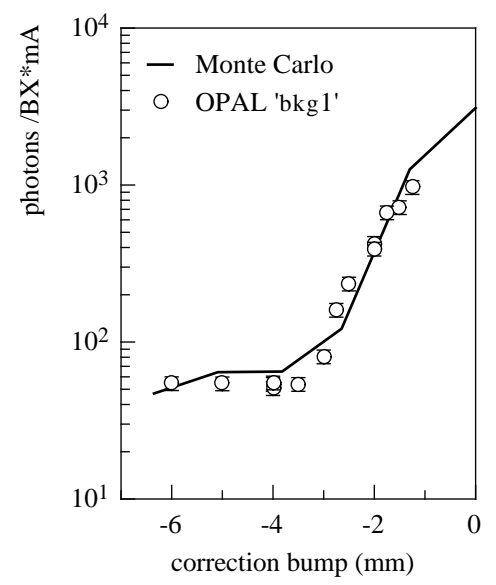

Figure 22: Comparison of measured and simulated photon rates in OPAL as function of the amplitude of an antisymmetric correction bump.

\subsubsection{OfF-ENERGY ELECTRON BACKGROUND WITH BUNCH TRAINS.}

The vertical separation bumps for head-on colliding bunch trains change the characteristics of the off-energy electron background at the luminosity detectors of 


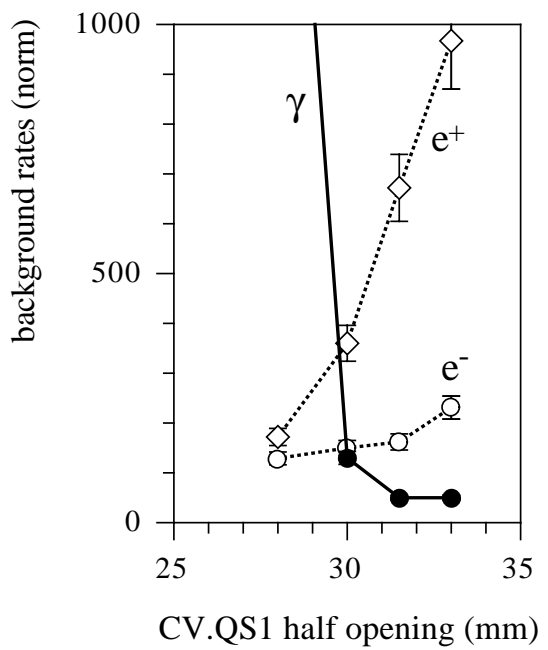

Figure 23: Measured background rates in OPAL as a function of the collimator CV.QS1 opening with 75\% separation bump. Off-energy particles (dotted lines), SR-photons (solid line).

the experiments. Although the absolute rate per $\mathrm{mA}$ stays about the same, there is a major shift of electron background from the horizontal into the vertical plane. The new vertical background family, first detected by OPAL during the $1995 \mathrm{Z}^{0}$ running [51], has very distinct characteristics: a sharp energy peak at $30 \mathrm{GeV}$ and a concentration in the vertical plane at small radii. These characteristics made it increasingly difficult for OPAL to discriminate off-energy particles from Bhabha events. It was shown experimentally that this background could be considerably reduced by the vertical collimator COLV.QS1, however at the cost of increasing the SR photon background rate (see Fig. 23).

Detailed MC simulations [41] showed that the effect was due to bremsstrahlung particles from upstream of the experiment which are directed into the vertical sectors of the luminosity detectors by the separation bump. All the observed features of this background [52] could be confirmed by simulation. The changes in the off-energy particles energy distribution with and without separation bump are shown in Fig. 24. The separation bump leads to a higher concentration of offenergy particles at small radii and into two distinct energy bands peaked around $30 \mathrm{GeV}$ into the lower detector, and around $15 \mathrm{GeV}$ into the upper detector. The origin of particles in the high momentum band is the region upstream of the downwards pointing first part of the separation bump. The $15 \mathrm{GeV}$ band originates from a region, much closer to the IP, along the upwards pointing part of the bump.

$\mathrm{MC}$ and experiment have shown that the disturbing $30 \mathrm{GeV}$ band can effec- 


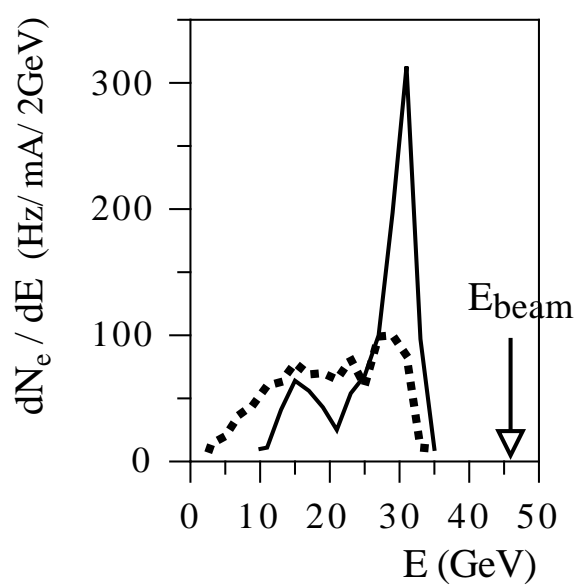

Figure 24: Simulated energy distribution for off-energy particles at the OPAL luminosity detectors. 100\% separation bump (solid line), no bump (dotted line).

tively be reduced by closing the nearest vertical collimators COLV.QS1 to below $30 \mathrm{~mm}$. However, this asks for a simultaneous reduction of the separation bump amplitude to $70 \%$ of its maximum value in order to avoid the high SR photon background from COLV.QS1. During the second part of the 1995 energy scan, this solution was adopted, and gave the expected improvement. Similar observations have been made by DELPHI during the $68 \mathrm{GeV}$ run at the end of 1995 [42]. As the inner acceptance for Bhabha electrons is at larger radius in the DELPHI luminosity calorimeter than it is in OPAL, this background does not enter the sample of Bhabha events. However it was found that their neutral trigger, which provides electron-photon separation, was very sensitive to the vertical off-energy background.

\subsection{Tests of the Synchrotron Radiation Masks}

Special experiments were performed in 1994 and 1995 to test the functionality of the SR-masks and to verify the quality of the Monte Carlo predictions. Although the large horizontal emittance expected at LEP2 cannot be simulated with LEP1 conditions, the number of small angle backscattered photons, that arrive at the beam-pipe without mask, can be enhanced by opening up the near horizontal collimators COLH.QS1. However, this also opens a window for direct photons from distant quadrupoles to reach the detectors.

From 1994 onward there were several studies devoted to comparing background in experiments with and without the mask. Here we describe the results from two major studies, one in August 1994 at $45 \mathrm{GeV}$, and the other in Novem- 
ber 1995 at $65 \mathrm{GeV}$. Both studies were made in physics conditions, i.e. colliding beams and collimators closed to their settings for physics data-taking.

We summarise briefly the results from the former, giving more detail from the latter which allowed comparison between the two experiments with the mask, DELPHI and OPAL, and the two without the mask, ALEPH and L3.

\subsubsection{The Background Measurements}

For both background studies the general strategy was to scan the horizontal collimator COLH.QS1 at $8.5 \mathrm{~m}$, opening and closing it from the nominal physics settings, thus enhancing the populations of direct and forward and backward scattered photons.

The first study in August 1994 [53] was made with Pretzel optics at a beam energy of $45.6 \mathrm{GeV}$. To avoid interactions of the 8 equally spaced bunches per beam at the arc mid-points, large horizontal "Pretzel orbits" are created over the entire length of the arcs.

The results were entirely satisfactory, with DELPHI being protected by the SRmasks from the small angle and direct SR-photons on opening COLH.QS1, while ALEPH, the experiment with the same machine optics as DELPHI, but without masks, suffered from the enhanced small angle and direct photons with their TPC consequently tripping. Fig. 25 shows data provided by the ALEPH small angle background monitor detector at two collimator openings, $25 \mathrm{~mm}$ and $50 \mathrm{~mm}$. At $25 \mathrm{~mm}$ one can see the superposition of signals from in-time electrons and photons and back-scattered photons from COLH.QS1. At the $50 \mathrm{~mm}$ opening these contributions are reduced by an order of magnitude, but one sees signals corresponding to the beam-pipe transition region at $55 \mathrm{~m}$, and from collimators COLV.QS5 $(90 \mathrm{~m})$ and COLH.QS6 $(110 \mathrm{~m})$. With the expected much larger beam emittance at LEP2 energies it is this photon family from the $55 \mathrm{~m}$ transition which will be strongly enhanced and would dominate the photon background without protection by SR-masks.

The study in November 1995 [54] was made with $4 \times 1$ on $4 \times 1$ bunch train optics at a beam energy of $65 \mathrm{GeV}$. This time the horizontal beam emittance could be increased to $50 \mathrm{~nm}$ using emittance wiggler magnets and by reducing the RF frequency. Vertical bunch train bumps in all four IP's were set to $20 \%$ of their maximum value, i.e. bump amplitudes stayed much below the critical threshold value, and no additional photon background resulted from these bumps.

Fig. 26 shows the background figure-of-merit "bkg1", normalised to a total current of $1 \mathrm{~mA}$, for all four experiments, and Fig. 27 shows photon hit rates from several detectors from ALEPH, DELPHI, and OPAL, all readings as a function of the COLH.QS1 opening and normalised to unity at the $30 \mathrm{~mm}$ collimator opening.

At openings beyond $45 \mathrm{~mm}$ the photon backgrounds rose sharply in the experiments without masks, ALEPH and L3, while a much smaller increase was observed in DELPHI and OPAL, equipped with the masks. For openings larger than $40 \mathrm{~mm}$ 

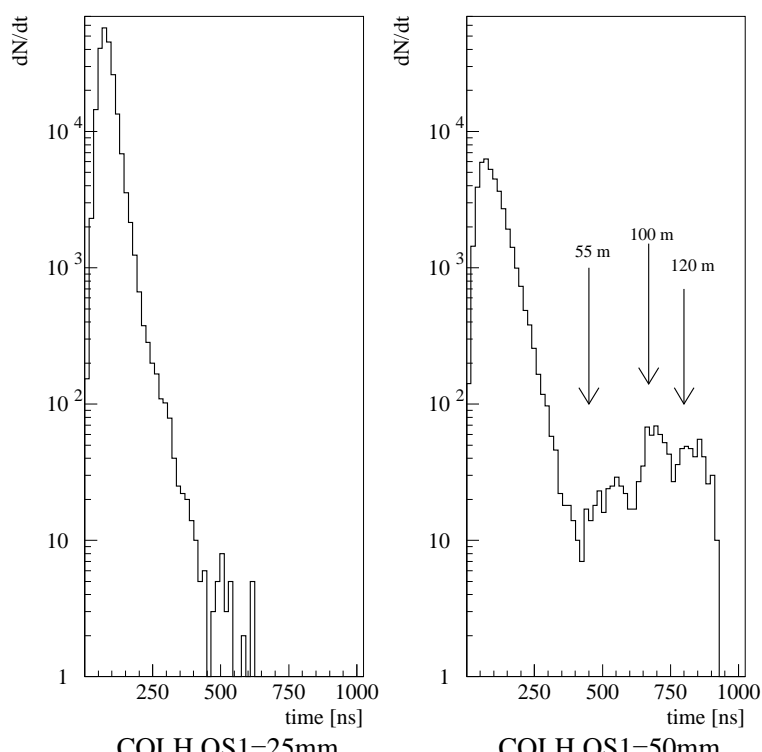

Figure 25: The distribution of arrival times of photons as observed in the ALEPH small angle background monitor for two half openings of the COLH.QS1 collimator. Backscattered photons which originate at distant beam elements are visible along with prompt photons.

the OPAL masks are hit by large numbers of direct photons, some of which are scattered back into the detectors. Probably due to the different optics in the IP8 region, this is not seen in DELPHI. A large fraction of the direct photons incident on the OPAL masks can be intercepted by the new collimator COLH.QS3. The dotted line in Fig. 27 gives the rate with COLH.QS3 completely retracted. During 1995 this collimator was only available in IP6. At small openings all experiments saw the enhanced background from large angle photons scattered from the collimator jaws, which the masks cannot protect against.

This study at $65 \mathrm{GeV}$ confirmed the importance of the synchrotron radiation masks which provide protection against photons reaching the central beam pipe at small angles.

\subsubsection{Comparison with Monte Carlo Simulations}

Monte Carlo simulations were carried out for both studies described above.

For the test at $45 \mathrm{GeV}$, Fig. 28 shows for DELPHI the comparison of Gaussian and exponential tail models with hit rates seen in the DELPHI TPC detector. It seems that some non-gaussian tails are needed to fit the measured hit rates at collimator openings above $35 \mathrm{~mm}$. Similar results were obtained for ALEPH.

For the test at 65 Gev Fig. 29 shows the comparison of measurements and 


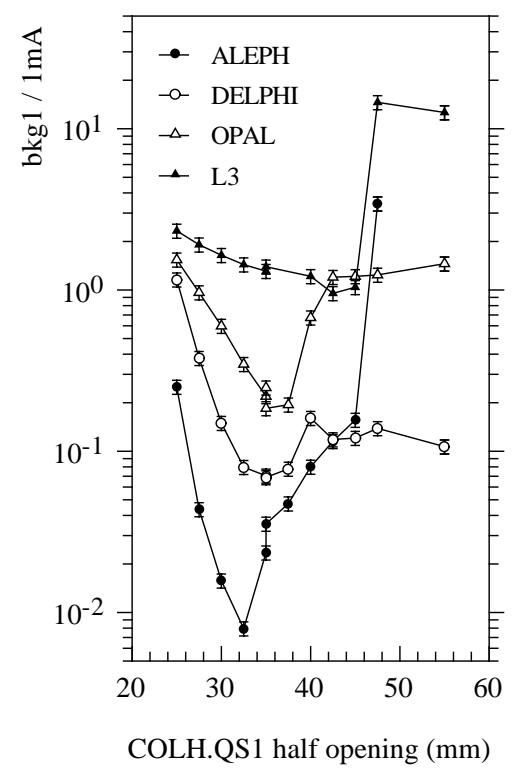

Figure 26: The figure-of-merit "bkg1" normalized to a total current of $1 \mathrm{~mA}$ as a function of the collimator setting.

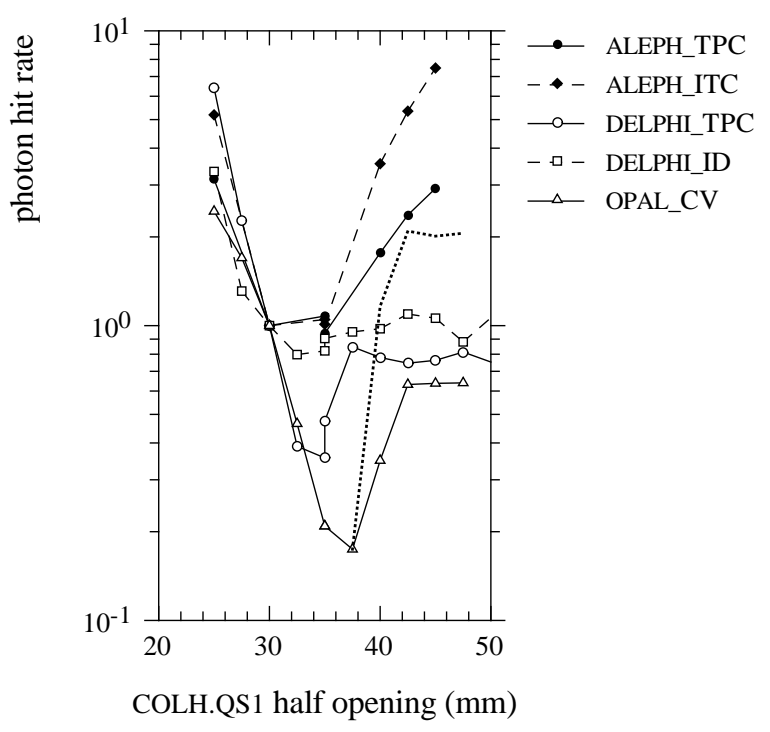

Figure 27: Photon hit rates for detectors as a function of the COLH.QS1 setting. The dotted line is the rate in the OPAL CV with open collimator COLH.QS3. 


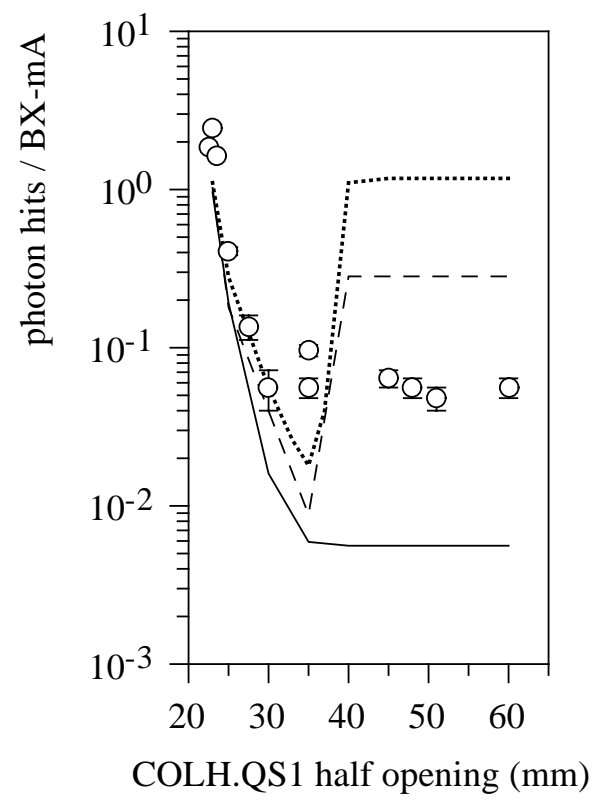

Figure 28: Monte Carlo predictions and photon hit rates in the DELPHI TPC at $45 \mathrm{GeV}$. The Monte Carlo code [6] was used with different assumptions for the density distributions of the electron beams: Gaussian (full line), Gaussian plus exponential tails above $4.5 \sigma_{x}$ (broken line) and Gaussian plus exponential tails above $4 \sigma_{x}$ (dotted line).

MC for the DELPHI TPC detector. Results are shown from the two different MC programs [6][7], that are used in this study. In both cases the absolute simulated photon rates incident at the DELPHI beam pipe, normalized for one crossing of two bunches with $500 \mu \mathrm{A}$ each, are folded with the detection probabilities into the TPC detector of Table 5, in order to give the simulated photon hit rates per BX-mA drawn in Fig. 29. This shows a remarkably good agreement between the two MC's and with the data. In order to get a good prediction for the SR photon background it is essential to model machine and beam conditions as closely as possible. This is particularly true for the horizontal beam emittance. During the $65 \mathrm{GeV}$ study, emittances were measured with $5 \%$ to $10 \%$ accuracy at every step in the collimator scan, and a current-weighted average of the horizontal emittances of the two beams have been used in the MC for each point. It is more difficult, but equally important for the modeling of SR background, to obtain information on the beam density distribution in the transverse planes. In the MC of Fig. 29 a purely Gaussian distribution was used in accordance to tail scans made during the MD [54]. In the region of collimator openings above 40 
$\mathrm{mm}$, where direct photons start to reach the edge of the downstream SR-masks, the resulting background rate is very sensitive to the density distribution in the tails. A $5 \%$ change in the horizontal emittance changes the predicted photon hit rate by a factor of five.

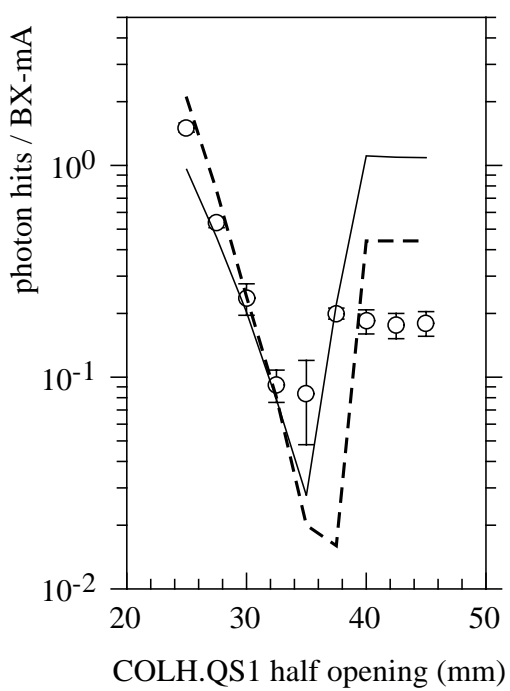

Figure 29: Monte Carlo predictions and measurements in the DELPHI TPC. Data points are from the DELPHI TPC, solid line MC [7], broken line MC [6]. $E_{\text {beam }}=$ $65 \mathrm{GeV}$ and the nominal horizontal emittance $\epsilon_{x}=50 \mathrm{~nm}$.

A similar comparison of measured and simulated photon hit rates in the OPAL CV detector shows the same behavior, see Fig. 30 .

\section{$5 \quad$ Expectations at High Energy}

\subsection{LEP2 Parameters}

The nominal LEP2 energy is $90 \mathrm{GeV}$ per beam, well above the production threshold of pairs of $\mathrm{W}$ bosons. However, with the total number of 272 superconducting cavities installed in 1998, the operational energy will reach $96 \mathrm{GeV}$ with an expected maximum peak luminosity of $1.4 \times 10^{32} \mathrm{~cm}^{-2} \mathrm{~s}^{-1}$ [55]. This high luminosity will be achieved by operating LEP in the bunch train mode [56] with four trains of two bunches per beam. The nominal betatron functions at the IP's are $\beta_{x}^{*}=1.25 \mathrm{~m}$ and $\beta_{y}^{*}=0.05 \mathrm{~m}$. Bunch currents of $0.5 \mathrm{~mA}$ are envisioned.

For a fixed magnetic and optical configuration the natural horizontal beam emittance $\epsilon_{x}$, increases with the square of the beam energy as $\epsilon_{x}$ is determined by the equilibrium between radiation damping and quantum excitation, both rapidly increasing with energy. At LEP2 the natural horizontal emittance is four times 


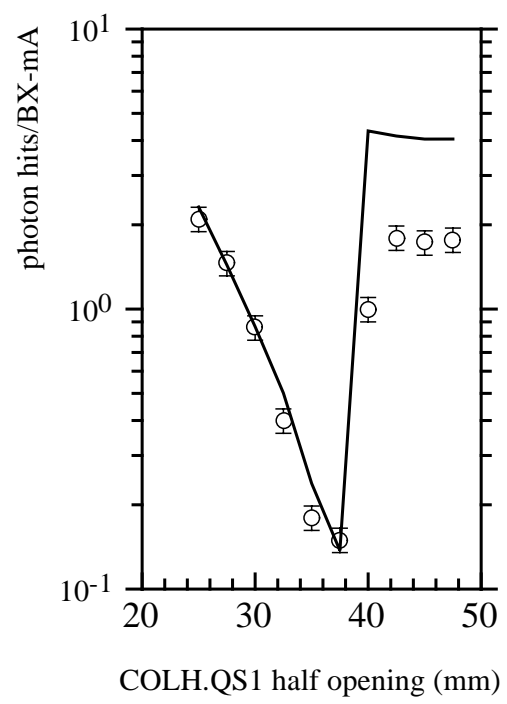

Figure 30: Monte Carlo predictions [7] (solid line) and measurements in the OPAL $\mathrm{CV}$ at $65 \mathrm{GeV}$.

larger than at LEP1 and reaches $\epsilon_{x}=46.0 \mathrm{~nm}$ at $90 \mathrm{GeV}$. This value holds for the nominal LEP2 optics with a phase advance per arc-cell of $90^{\circ}$ and $60^{\circ}$ in the horizontal and vertical planes, respectively. A low emittance optics with stronger focusing in the horizontal plane $\left(\Delta \mu_{x}=108^{\circ}\right)$ has been proposed for LEP2, which would reduce the horizontal emittance by a factor of $2 / 3$. However, the dynamic aperture has been shown by beam tests to be much more restricted than had been expected.

The horizontal emittance can also be reduced by increasing the horizontal damping partition number $J_{x}$ from its nominal value $J_{x}=1$. $J_{x}$ can be increased by a small reduction of the RF frequency. An increase of $J_{x}$ from 1 to 1.5 will reduce the $90 \mathrm{GeV}$ horizontal emittance from $46.0 \mathrm{~nm}$ to $30.7 \mathrm{~nm}$ [55]. This is a realistic option for the high energy operation of LEP.

\subsection{Off-energy background}

Off-energy particle background at LEP predominantly stems from beam-gas bremsstrahlung along the straight sections. The cross-section of this process is independent of beam energy to first order. The rate of off-energy particles is proportional to the vacuum pressure and beam current. It rises, however, with the square of the current if the gas pressure due to synchrotron radiation outgassing (dynamic pressure) is significant. This is expected at higher beam energy and has been observed by the LEP experiments. 
Measurements of the dynamic pressure in straight sections at different energies have been reported [57]. When compared to the well cleaned machine at $45 \mathrm{GeV}$ the measured value of the dynamic pressure, averaged over the four even straight sections, has increased by a factor of 5 at $65 \mathrm{GeV}$ in 1995 . The dynamic pressure is expected to further increase with beam energy, however the exact behaviour is not known.

\subsection{Synchrotron radiation}

Photon background from synchrotron radiation rises sharply with beam energy with the dominant factor being the increasing $\left(\propto E^{2}\right)$ horizontal beam emittance. Expected SR-photon rates incident at the beam pipe in the experiments at high energy have been simulated using the MC code [7]. Although the measured SR photon rates strongly depend on many different parameters, e.g. optics, beam dynamics, and the background protection system, a comparison of measured and simulated photon rates under well controlled beam conditions gave sufficiently good agreement (chapter 4.5) to rely on MC for the estimation of photon background levels a higher beam energies in LEP.

Simulations have been performed for the $\Delta \mu_{x / y}=90^{\circ} / 60^{\circ}$-optics and with nominal beta values at the IP's of $\beta_{x}^{*}=1.25 \mathrm{~m}$ and $\beta_{y}^{*}=0.05 \mathrm{~m}$. However, due to SC cavity installation requests, two different types of straight section optics are required for LEP2. Optics in IP2 and IP6 are different from those in IP4 and IP8, which can have important consequences for the SR-photon background. The natural horizontal emittance is scaled for energy according to

$$
\epsilon_{x}(E)=46.0[\mathrm{~nm}](E[\mathrm{GeV}] / 90)^{2}
$$

and the vertical emittance is assumed to be $\epsilon_{y}=0.05 \epsilon_{x}$. A safety factor of 1.2 is applied to the natural emittances. Gaussian density distributions of the beams in the horizontal plane and exponential distributions in the vertical plane are assumed. At $45.6 \mathrm{GeV}$ a blown-up emittance of $40 \mathrm{~nm}$ is used. Under these conditions the total radiated SR power in the IP4 straight section quadrupoles rises with $E^{4}$ and the number of radiated photons with photon energy $E_{\gamma}>10 \mathrm{keV}$ increases as $E^{2.2}$. It reaches a level of $1.4 \times 10^{12}$ photons/BX-mA at $90 \mathrm{GeV}$.

As described in chapter 2.4.2 the main contribution to the SR-photon background at high beam energies and large emittances is due to small angle photons back scattered from beam pipe elements some $50 \mathrm{~m}$ downstream of the IP. This family can be almost completely suppressed by installing SR-masks. The remaining background from large angle photons is due to scattering from the nearest collimators and cannot be intercepted by the masks. However, about half of this contribution, the forward scattered part, is eliminated by the design of the new COLH.QS1 collimators.

Simulated photon rates versus beam energy for the two cases with and without LEP2 protection are shown in Fig. 31. The efficiency of the SR-masks plus 
additional LEP2 protection elements to shield the experiments against the exponentially increasing rate of SR photons with energy is evident. Reduction factors of two to three orders of magnitude are obtained. The photon level seen at $45 \mathrm{GeV}$ without mask, where the horizontal emittance was blown up to $40 \mathrm{~nm}$ in order to avoid destructive beam-beam interactions, is only reached again at about $95 \mathrm{GeV}$ with the LEP2 protection active. At $100 \mathrm{GeV}$ the expected rate is 2.3 times above the $45 \mathrm{GeV}$ level. A reduction of the horizontal emittance by $20 \%$, i.e. to its nominal value, and closing collimators to $12 \sigma_{x}$ instead of $14 \sigma_{x}$, reduces the expected photon rates at high energies by a factor of about 4 .

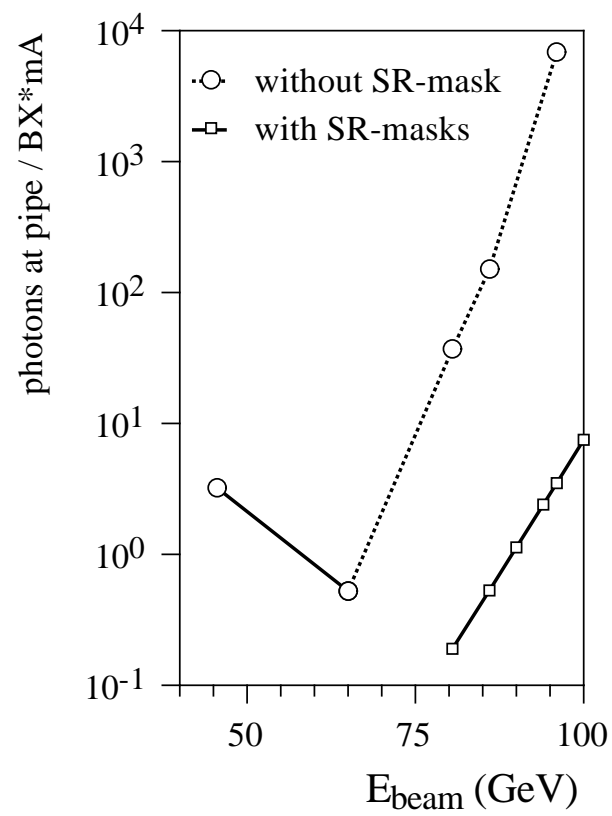

Figure 31: Simulated SR photon rates in IP4 versus beam energy with and without LEP2 protection (with and without SR-mask). The described beam conditions are used with an additional safety factor of 1.2 on the horizontal emittance. Aperture limiting collimators are closed to $12 \sigma_{x}$ and $30 \sigma_{y}$. All collimators are set at their optimum openings within the above limits.

The simulations of Fig. 31 assume however that the SR-masks are completely protected against hits of direct photons that are radiated in upstream straight section quadrupoles. This protection is provided by the special LEP2 collimators COLH.QS3 and the quality of protection is dependent on the optics parameters at the location of these collimators and the beam emittance. Simulated photon rates as function of the collimator COLH.QS3 opening at the two types of IP's, having 
different optics, are shown in Fig. 32. A specific high energy $\left(90^{\circ} / 60^{\circ}\right)$-optics with $\beta_{x}^{*}=2 \mathrm{~m}$ and nominal emittances at $92 \mathrm{GeV}$ is used for these calculations. With COLH.QS3 completely open up to $10^{8}$ photons/BX-mA can reach directly the tip of the downstream mask from where they are back scattered into the experiment with a probability of several $10^{-5}$. Due to different straight section optics, nearly two orders of magnitude more photons reach the mask in IP2 than in IP4. The mask can be effectively shielded, reducing the photon background rate by 4 to 6 orders of magnitude, if collimator COLH.QS3 can be closed to $15 \mathrm{~mm}$. With apertures set to $12 \sigma_{x}$, the collimator cannot be closed to below $14 \sigma_{x}$, if scraping into the beam tails is to be avoided. With the optics chosen the beam size at COLH.QS3 in IP2 (and IP6) is sufficiently small to allow for a complete protection of the SR-masks, while only incomplete protection is possible in IP4 (and IP8).

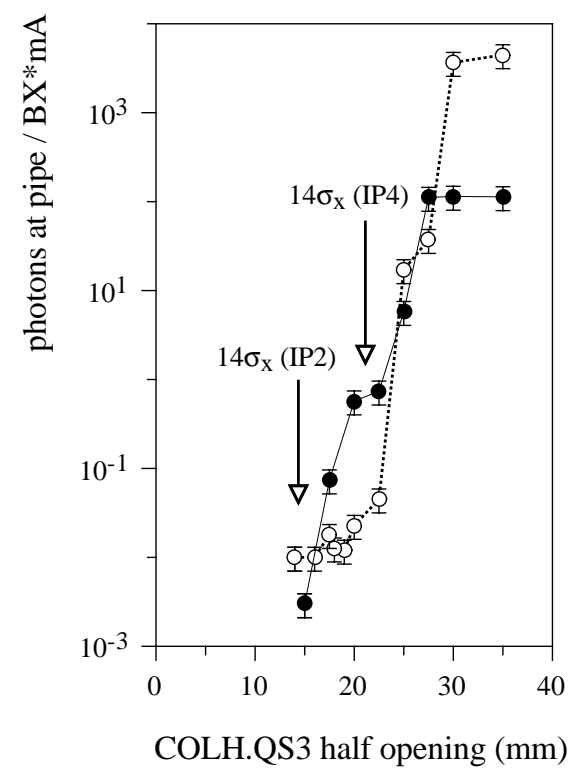

Figure 32: Simulated photon rate incident at the beam pipes in IP2 (open circles) and IP4 (full circles) as function of the collimator COLH.QS3 opening. $E_{\text {beam }}=$ $92 \mathrm{GeV}, \epsilon_{x}=48.1 \mathrm{~nm}$, aperture collimators at $12 \sigma_{x}$.

While photon rates in the two latter interaction points, for the pessimistic assumptions of the beam emittances with $J_{x}=1$, are still reasonably small, the importance of the straight section beam optics at high energies with the SRmasks in place is evident. If $J_{x}$ values of order 1.5 can be reached during high energy physics running, thereby reducing the horizontal emittance at $92 \mathrm{GeV}$ to $31 \mathrm{~nm}$, full protection is also possible in IP4 and IP8. The same result is obtained if the low emittance optics with increased horizontal phase advance of $108^{\circ}$ per 
cell can be used. In these cases the expected SR-photon background rates at energies above $90 \mathrm{GeV}$ should be quite acceptable in all four experiments.

If at very high beam energies the SR-photon background rates should become intolerably high, specific modification of the straight section optics, aimed at restoring full protection of the SR-masks, could be envisaged.

\section{Conclusion}

The very rapid increase of synchrotron radiation photon background at the LEP experiments expected with higher beam energies for LEP2 requires a considerable strengthening of the background protection system in LEP.

Simulations of photon background have been performed, using the "PHOTON" code and the results are compared with measurements taken by the four experiments for well defined machine conditions at $45.6 \mathrm{GeV}$ and $65 \mathrm{GeV}$. The good agreement obtained, gave confidence to base the design of an upgrade of the LEP2 background protections system on PHOTON predictions at high energies.

The heart of the new LEP2 protection are tungsten absorber rings, 'SR-masks', installed about $2.5 \mathrm{~m}$ from the IP inside the experimental vacuum pipes. Further additions are enlarged vacuum chambers between $25 \mathrm{~m}$ to $65 \mathrm{~m}$ from the IP's on either side of the experiments, additional as well as improved movable tungsten collimators and photon absorbers.

First measurements with part of this system installed during 1995 and 1996 showed the correct functioning of the new protection. The full complement will be present for the 1997 physics run at $92 \mathrm{GeV}$ per beam. Expected photon rates at energies above $90 \mathrm{GeV}$ with the full LEP2 protection in place are of the same order as were seen during physics data taking at $45 \mathrm{GeV}$ with LEP1.

However, rates can rapidly rise above this level if the SR-masks cannot be completely protected against impacts from photons that are radiated in far upstream quadrupole fields and reach the tip of the downstream masks directly. Masks are protected against these direct photons by a pair of new collimators placed close to a horizontal beam waist some $50 \mathrm{~m}$ away on either side of the IP. The quality of this protection however depends on the specific optics installed in the straight section and is weakened with larger horizontal beam emittances or non-gaussian beam tails. Predictions at $92 \mathrm{GeV}$, for pessimistic assumptions of the beam size and for realistic straight section optics show that the SR-masks can still be sufficiently protected.

If at very high energies photon background fluxes should become too high, specific modifications of the optics, aimed at improved protection of SR-masks, might be the only remedy.

Background rates of off-energy electrons are independent of beam energy to first order. However, they grow with the square of the beam current, rather than linearly, if the dynamic vacuum pressure at high energies substantially contributes 
to the average vacuum pressure along the straight sections. The dynamic pressure does increase with energy due to increased synchrotron radiation, however the exact behaviour is not known.

\section{Acknowledgements}

We would like to thank our colleagues in the four LEP experiments and the SL Division of CERN for making these studies possible. The important contributions of the LEP Operations Team by setting-up and controlling the LEP accelerator under the special conditions required for many of our MD experiments are gratefully acknowlegded. GvH would like to warmly thank Claude Bovet for his constant support and interest shown over many years of this work and for many fruitful discussions. 


\section{References}

[1] J. M. Jowett, "More bunches in LEP," 1989 Particle Accelerator Conference, Chicago, IL, (1989).

[2] R. Bailey et al, "Studies on Bunch Trains in LEP", Europ. Part. Acc. Conf.(EPAC94), London, 1994.

[3] W. Herr, "Bunch Trains without Crossing Angle", proceedings of the 4th Workshop on LEP Performance, Chamonix, 1994.

[4] Particle Data Group, Phys. Lett. B239 (1990)

[5] J. Schwinger, Phys. Rev. 75 (1979) 1912.

[6] P. Roudeau, (unpublished).

[7] P. Münger and G. von Holtey, 1985, (unpublished).

[8] G. von Holtey and K.M. Potter, "Estimation of the Synchrotron Radiation Background in LEP Experiments", IEEE Part. Acc. Conf., Washington, DC, 1987

[9] K. L. Brown and C. Iselin, "Decay Turtle- A computer program for simulating charged particle beam transport systems, including decay calculations," CERN 74-2 (1974).

[10] H. Burkhardt, R. Kleiss; Beam Lifetimes in LEP, Proc. 4th European. Part. Acc. Conf. EPAC London 1994, Eds. V. Suller and Ch. Petit-Jean-Genaz, Vol II page $1353-1355$

[11] R. Kleiss, H. Burkhardt, CERN-SL/94-03 (OP) and NIKHEF-H-94-01 BBBREM: Monte Carlo Simulation of Radiative Bhabha Scattering in the very forward direction. Comp. Phys. Comm. 81:372-380,1994

[12] V. I. Telnov, Nucl. Instr. and Methods A260 (1987) 304.

[13] B. Dehning, A. C. Melissinos, F. Perrone, C. Rizzo and G. von Holtey, Phys. Lett. B249 (1990) 145.

[14] H. Burkhardt, Monte Carlo Simulation of Beam Particles and Thermal Photons; CERN/SL Note 93-73 (OP), 1993 (unpublished).

[15] Yung-Su Tsai, Pair production and bremsstrahlung of charged leptons, Rev. Mod. Phys 46 No.4 (1974) 815

[16] O.Gröbner, Vacuum performance, in LEP Design Report Vol. III, LEP2, CERN AC/96-01 (1996).

[17] H. Burkhardt et al. Beam Tails in LEP. In V. Suller and Ch. Petit-JeanGenaz, editors, Proceedings of the 5th European Particle Accelerator Conference, volume 2, pages 1152-1154, 1996.

[18] J. Rothberg, "Limitations due to Backgrounds at LEP1", Proc. 5th Workshop on LEP Performance, Chamonix, 1995. 
[19] G. von Holtey, "Electron Beam Collimation at LEP Energies", IEEE Part. Acc. Conf., Washington, DC, 1987

[20] F. Bertinelli and R. Jung, "Design and Construction of LEP Collimators", IEEE Part. Acc. Conf., Washington, DC, 1987

[21] G. von Holtey, S. Meyers and A. Verdier, "Proposal for a Physics Optics with $\beta_{x}^{*}=2.5 \mathrm{~m} "$, SL/Note 92-60 1992, (unpublished).

[22] G. von Holtey, "Simulations of Photon Backgrounds to LEP Detectors at 90 GeV Beam Energy", CERN SL/92-54 (1992)

[23] The DELPHI Collaboration, "Proposal for the upgrade in the forward region," CERN/LEPC 92-13 Add 2, 16 October 1992.

The LEPC Working Group on LEP2 Backgrounds, "Proposal for a Synchrotron Radiation Test Mask in the DELPHI Detector", CERN/LEPC9310 (1993).

[24] H. Kichimi et al, "Mask System for the TOPAZ Detector at TRISTAN", Nucl. Instr. and Methods, A334 (1993) 367.

[25] S. Herb, private communication.

[26] G. von Holtey, W. Niessen, P Roudeau, "Synchrotron Radiation Masks for LEP2", 4th European Part. Acc. Conf., EPAC94, London (1994)

[27] F. Harris, "Particle backgrounds and synchrotron radiation masks at $90 \mathrm{GeV}$ ", Proceedings 5th Workshop on LEP Performance, Chamonix,1995.

[28] ALEPH: D. Decamp et al, Nucl. Instr. and Methods, A 294 (1990) 121.

[29] Delphi: P. Aarnio et al, Nucl. Instr. and Methods, A 303 (1991) 233.

[30] L3: B. Adeva et al, Nucl. Instr. and Methods, A 289 (1990) 35.

[31] OpAL: K. Ahmet et al, Nucl. Instr. and Methods, A 305 (1991) 275.

[32] A. Schwarz, "Heavy Flavour Physics at Colliders with Silicon Strip Vertex Detectors," Physics Reports 238 (1994) 1.

[33] R. Johnson and R. Rennels, "Radiation Damage Measurements on ALEPH Double-sided Silicon Strip Test Structures," ALEPH 94-099. June 1994, (unpublished).

[34] J. Kadyk, "Wire Chamber Aging", Nucl. Instr. and Methods, A 300 (1991) 436.

[35] S. Ahlen et al, Nucl. Instr. and Methods, A 369 (1996) 92.

[36] GEANT, Detector Description and Simulation Tool, CERN Program Library, W5013.

[37] A.C. Benvenutti et al, "DELPHI Background Measurements at $65 \mathrm{GeV}$ ", DELPHI 96-13 LEDI 6, 23 Jan 1996, (unpublished).

[38] M. Hauschild, private communication, 1996. 
[39] H. Burkhardt, R. Jacobsen, G. Lutters,, and G. von Holtey, "Effects of Aperture Limiting Collimators," CERN SL-MD Note 111, February 1994, (unpublished).

[40] H. Burkhardt, G. von Holtey and A. H. Ball, "Particle Background at LEP with Head-on Colliding Bunch Trains", Proc. 5th Europ. Part. Acc. Conf., EPAC96 (1996)

[41] G. von Holtey, "Off-Energy Electron Background with Bunch Trains in LEP", CERN SL/95-86, October 1995.

[42] E. Falk, V. Hedberg, G. von Holtey, "Simulation of off-energy Electron Background in DELPHI," CERN SL/97-04, February 1997.

[43] G.S. Brown and D.E. Moncton, Handbook on Synchrotron Radiation, Volume 3, Amsterdam: North-Holland, 1991, pp 120-124.

[44] J. Rothberg and J. Wear, "Main Observations from MD of 8 September 1993", 13 September 1993, (unpublished).

[45] G. von Holtey, "Synchrotron Radiation Photon Background with Bunch Trains in LEP", CERN SL/95-29, July 1995.

[46] The LEPC Working Group on Machine induced Particle Backgrounds at LEP2 Energies, J. Rothberg et al, "Dependence of backgrounds at the LEP detectors from orbit amplitudes", CERN SL-MD Note 85, June 1993, (unpublished).

[47] G. von Holtey, "Monte Carlo Simulations of Photon Background Measurements during the June 1993 MD", CERN SL-MD Note 97, August 1993, (unpublished).

[48] C. Bovet et al, "Final Report of the 1994 Bunch Train Study Group", CERNSL/94-95, Dec.1994

[49] W. Herr, G. von Holtey, A. Spinks and J. Wenninger, "Study of Photon Background with Bunch Trains", CERN SL-MD Note 159, December 1995, (unpublished).

[50] H. Burkhardt, W. Herr, G. von Holtey, The ALEPH, DELPHI, L3 and OPAL Collaborations, "Measurements of the Photon Background at LEP Detectors with Symmetric and Asymmetric Vertical Bumps for Bunch Trains", CERN SL-MD Note 143, September 1995, (unpublished).

[51] A. H. Ball and R. Kelogg, private communication, OPAL 1995

[52] P. Estabrooks, M. Fanti and P. Weber, "Beam-induced Backgrounds in OPAL: A study of LEPI conditions and projections for LEPII", OPAL Technical Note TN292, March 1995, (unpublished).

[53] K. Bell et al, "Test of Synchrotron Radiation Masks in LEP", CERN SL-MD Note 144, 1994, (unpublished). 
[54] A. Ball et al, "Measurement of Particle Backgrounds at the LEP Experiments with $65 \mathrm{GeV}$ Beams", CERN SL-MD Note 201, 1996 (unpublished).

[55] LEP Design Report, Vol. III LEP2, CERN-AC/96-01 (LEP2).

[56] C. Bovet, O.C. Brunner, B. Goddard, W. Herr, G. von Holtey, E. Keil, M. Lamont, M. Meddahi, E Peschardt, J. Poole, "Report of the 1995 BunchTrain Study Group", CERN-SL/96-12 (AP), 1996.

[57] N. Hilleret, private communication, 1996. 


\section{A Collimators and Masks}

Tables A.1 and A.2 give the properties of the LEP collimators.

Table A.1: LEP background collimators ( $\mathrm{H}=$ horizontal plane, $\mathrm{V}=$ vertical plane); 1995 layout, settings for $45.6 \mathrm{GeV}$.

\begin{tabular}{|l|cccc|}
\hline $\begin{array}{l}\text { collimator } \\
\text { name }\end{array}$ & $\begin{array}{c}\text { IP2, IP6 } \\
\text { distance }[\mathrm{m}]\end{array}$ & $\begin{array}{c}\text { IP4, IP8 } \\
\text { distance [m] }\end{array}$ & type & nominal setting \\
\hline COLH.QS1 & 8.5 & 8.5 & SR (near),H & $\pm 16 \sigma_{h}$ \\
COLV.QS1 & 8.6 & 8.6 & SR (near),V & $\pm 32 \sigma_{v}$ \\
COLZ.QS2 & 21.3 & 21.3 & ZL, V & $\pm 35 \mathrm{~mm}$ \\
COLH.QS3 $^{a}$ & $56.3^{b}$ & & SR, H & $\pm 15 \sigma_{h}$ \\
COLZ.QS4 $^{b}$ & 66.1 & 66.1 & ZL, V & $\pm 40 \mathrm{~mm}$ \\
COLV.QS5 & 98.7 & 89.7 & SR (far), $\mathrm{V}$ & $\pm 40 \sigma_{v}$ \\
COLH.QS6 $^{\text {COLH.QS10 }}$ & 129.4 & 108.0 & SR (far),H & $\pm 15 \sigma_{h}$ \\
COLH.QS15 $^{a}$ & 220.5 & 220.2 & SR, H & $\pm 15 \sigma_{h}$ \\
COLH.QS17 $^{\text {COLH.QD20 }}{ }^{a}$ & 356.6 & 356.6 & off-E, H & $-13 \sigma_{h}$ \\
COLH.QF23 & 522.2 & 419.6 & off-E, H & $-13 \sigma_{h}$ \\
\hline
\end{tabular}

${ }^{a}$ added for LEP2

${ }^{b}$ IP6 only

Table A.2: LEP aperture collimators ( $\mathrm{H}=$ horizontal, $\mathrm{V}=$ vertical plane); 1995 layout, settings for $45.6 \mathrm{GeV}$.

\begin{tabular}{|l|ccc|}
\hline collimator & location & type & nominal setting \\
\hline COLH.IP5 & IP5 & aperture, H & $\pm 12.5 \sigma_{h}$ \\
COLH.QL13L,R & IP5 & aperture, H & $\pm 13.5 \sigma_{h}$ \\
COLV.QD30 & arc 5 & aperture, V & $\pm 25 \sigma_{v}$ \\
COLH.QD20,40 & arc 5 & aperture, V & $\pm 27 \sigma_{v}$ \\
\hline
\end{tabular}


The specifications of the SR masks and shield are given in Table A.3. The symbols used in the table are defined in Fig. A.1.

Table A.3: Specifications of SR masks and shield for the four LEP experiments.

\begin{tabular}{|l|c|cccc|}
\hline & Nominal & ALEPH & DELPHI & L3 & OPAL \\
\hline SR mask & & & & & \\
$L_{\text {mask }}[\mathrm{m}]$ & 2.30 & 2.42 & 2.46 & 2.60 & 2.55 \\
$R_{\text {mask }}[\mathrm{mm}]$ & 42.0 & 41.0 & 41.0 & 41.0 & 41.0 \\
$L[\mathrm{~mm}]$ & 100 & 100 & 100 & 100 & 90 \\
$\gamma[\mathrm{deg}]$ & 1 & 1 & 1 & 1 & 1 \\
$\alpha[\mathrm{deg}]$ & 15 & 45 & 15 & 45 & 45 \\
$\beta[\mathrm{deg}]$ & 15 & 20 & 15 & 20 & 20 \\
\hline Shield & & & & & \\
$\delta[\mathrm{mr}]$ & 30 & 34 & 40 & 30.6 & 33.5 \\
$L_{\text {shield }}[\mathrm{m}]$ & 1.83 & 1.93 & 1.94 & 2.02 & 1.94 \\
gap $[\mathrm{mm}]$ & 0 & 5 & 10 & 5 & 5 \\
$d[$ mm $]$ & 20 & 24 & 10 & 10 & 5 \\
\hline Shadow & & & & & \\
$\phi_{\text {shadow }}[\mathrm{mr}]$ & 2.66 & 2.75 & 2.73 & 2.60 & 2.67 \\
\hline
\end{tabular}

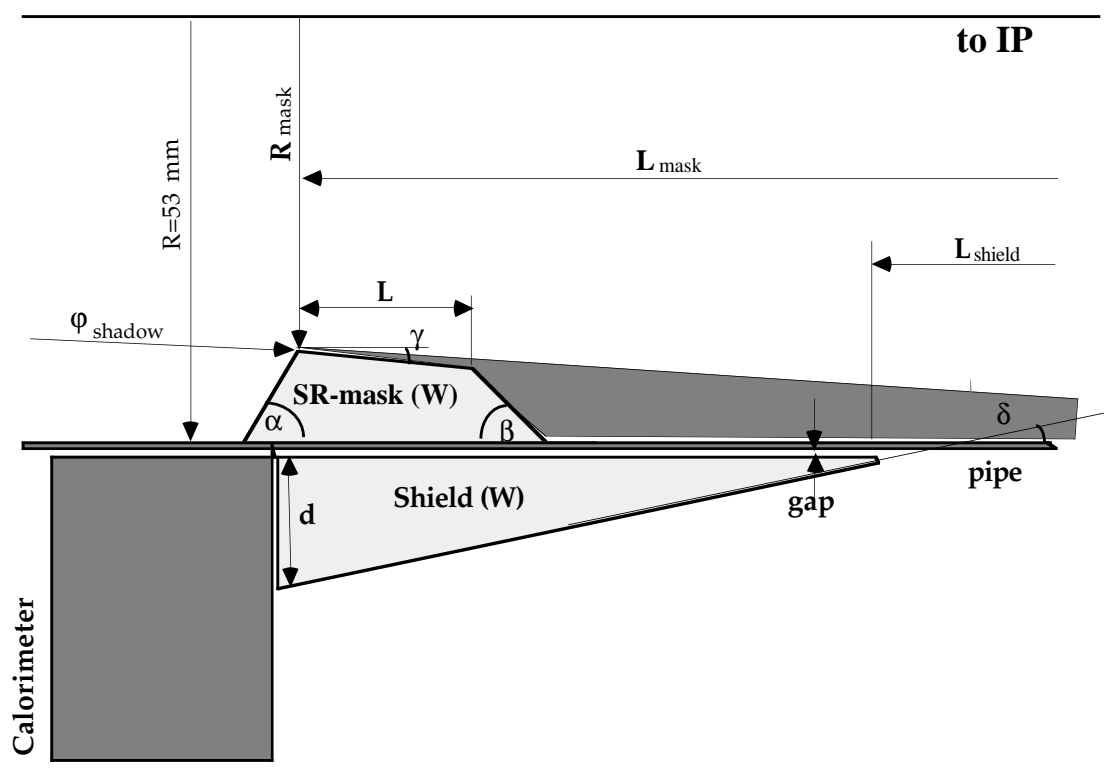

Figure A.1: Cut through cylindrical SR mask and outer shield 


\section{B Detector Properties}

Tables B.1, B.2, B.3, B.4, B.5, B.6 summarize the properties of the detectors in each of the LEP experiments which are most vulnerable to background. Tables B.7, B.8 list the detectors used specifically for background monitoring.

Table B.1: Properties of ALEPH subdetectors.

\begin{tabular}{|c|c|c|c|}
\hline & ITC & $\mathrm{TPC}$ & SCAL \\
\hline Type & Drift Chamber & $\mathrm{TPC}$ & W-Si Calorim. \\
\hline Purpose & Tracking,Trigger & Tracking & Luminosity \\
\hline Radial size & $128-280 \mathrm{~mm}$ & $0.3-1.8 \mathrm{~m}$ & $\begin{array}{c}60-150 \mathrm{~mm} \\
\theta=24-60 \mathrm{mrad}\end{array}$ \\
\hline Length & $\pm 1 \mathrm{~m}$ & $\pm 2.2 \mathrm{~m}$ & $124 \mathrm{~mm}$ \\
\hline Location & $\begin{array}{l}\text { IP } \\
8 \text { radial layers }\end{array}$ & $\begin{array}{c}\text { IP } \\
36 \text { sectors }\end{array}$ & $\pm 2.5 \mathrm{~m}$ \\
\hline Operating Cond & $1 \mathrm{Atm} \mathrm{Ar}-\mathrm{CO}_{2}$ & $1 \mathrm{Atm} \mathrm{Ar}-\mathrm{CH}_{4}$ & \\
\hline Sensitivity & $\begin{array}{l}\text { photons } \\
\gtrsim 1 \mathrm{keV}\end{array}$ & $\begin{array}{l}\text { photons } \\
\gtrsim 1 \mathrm{keV}\end{array}$ & $\begin{array}{l}\text { electrons } \\
\gtrsim 20 \mathrm{GeV}\end{array}$ \\
\hline $\begin{array}{l}\text { Background } \\
\text { Limits }\end{array}$ & current & $\begin{array}{c}\text { current } \\
\text { (100 nA/sector) }\end{array}$ & $\begin{array}{l}\text { off-energy electrons } \\
\text { singles trigger rate }\end{array}$ \\
\hline $\begin{array}{l}\text { affected by } \\
\text { background }\end{array}$ & $\begin{array}{l}\text { detector } \\
\text { lifetime }\end{array}$ & $\begin{array}{l}\text { detector } \\
\text { lifetime }\end{array}$ & $\begin{array}{l}\text { Luminosity } \\
\text { precision }\end{array}$ \\
\hline $\begin{array}{l}\text { background } \\
\text { data }\end{array}$ & $\begin{array}{l}\text { hit rate, current. } \\
\text { radial, angular, } z \\
\text { distributions. } \\
\text { time distributions } \\
\text { far sources } \lesssim 60 \mathrm{~m} \text {. }\end{array}$ & $\begin{array}{l}\text { hit rate, current } \\
\text { "bkg1"=current }\end{array}$ & not available \\
\hline
\end{tabular}


Table B.2: Properties of OPAL subdetectors.

\begin{tabular}{|c|c|c|c|}
\hline & $\mathrm{CV}$ & C.J & SW \\
\hline Detector Type & Vertex Drift Chamber & Jet Drift Chamber & Si-W Calorim. \\
\hline Normal purpose & Tracking,trigger & Tracking,trigger & Luminosity \\
\hline Radial size & $103-235 \mathrm{~mm}$ & $.25-1.85 \mathrm{~m}$ & $\begin{array}{l}62-142 \mathrm{~mm} \\
26-59 \mathrm{mrad}\end{array}$ \\
\hline Length & $\pm 50 \mathrm{~cm}$ & $\pm 2 \mathrm{~m}$ & \\
\hline Location & $\begin{array}{c}\text { IP } \\
18 \text { radial layers } \\
36 \text { sectors }\end{array}$ & $\begin{array}{c}\mathrm{IP} \\
159 \text { radial layers } \\
24 \text { sectors }\end{array}$ & $z= \pm 2.4 \mathrm{~m}$ \\
\hline Operating Cond & 4 Atm $\mathrm{Ar}-\mathrm{CH}_{4}-\mathrm{iC}_{4} \mathrm{H}_{10}$ & 4 Atm Ar- $\mathrm{CH}_{4}-\mathrm{iC}_{4} \mathrm{H}_{10}$ & \\
\hline Sensitivity & photons, electrons & photons, electrons & electrons \\
\hline $\begin{array}{l}\text { Background } \\
\text { Limits }\end{array}$ & $\begin{array}{l}\text { current } \\
\text { hit rate }\end{array}$ & current & $\begin{array}{l}\text { off-energy electrons } \\
\text { singles trigger rate }\end{array}$ \\
\hline $\begin{array}{l}\text { affected by } \\
\text { background }\end{array}$ & detector lifetime & detector lifetime & $\begin{array}{l}\text { Luminosity } \\
\text { precision }\end{array}$ \\
\hline $\begin{array}{l}\text { background } \\
\text { data }\end{array}$ & $\begin{array}{l}\text { hit rate, } \\
\text { radial, azimuthal } \\
\text { distributions. } \\
\text { time distributions } \\
\text { "bkg1" = hit rate }\end{array}$ & & $\begin{array}{c}\text { "bkg2" = } \\
\text { Acc./ Bhabha rate }\end{array}$ \\
\hline
\end{tabular}


Table B.3: Properties of L3 subdetectors.

\begin{tabular}{|c|c|c|c|}
\hline & TEC & $\overline{\mathrm{FTC}}$ & LUMI \\
\hline Type & $\begin{array}{c}\text { Time Expansion Chamber } \\
\text { Drift chamber }\end{array}$ & $\begin{array}{c}\text { Forward Tracking Chamber } \\
\text { Drift chamber }\end{array}$ & $\begin{array}{l}\text { BGO Calorimeter } \\
+ \text { Si tracker }\end{array}$ \\
\hline Purpose & Tracking & $\begin{array}{c}\text { Tracking } \\
\text { Bkg. Monitor }\end{array}$ & $\begin{array}{c}\text { Luminosity } \\
\text { Measurement }\end{array}$ \\
\hline $\begin{array}{l}\text { Location } \\
\text { Size }\end{array}$ & $\begin{array}{c}r=85 .-468 \mathrm{~mm} \\
\pm 0.5 \mathrm{~m} \text { Long } \\
8+52 \text { radial layers }\end{array}$ & $\begin{array}{c}120-490 \mathrm{~mm} \\
\mathrm{z}= \pm 65 \mathrm{~cm} \\
20(\mathrm{H})+20(\mathrm{~V}) \text { sectors }\end{array}$ & $\begin{array}{c}68-190 \mathrm{~mm} \\
\pm 265 \mathrm{~cm} \text { from } \\
\text { interaction point }\end{array}$ \\
\hline $\begin{array}{l}\text { Operating } \\
\text { Conditions }\end{array}$ & $\begin{array}{c}1.2 \mathrm{Bar} \\
\mathrm{CO}_{2}-\mathrm{iC}_{4} \mathrm{H}_{10}\end{array}$ & & \\
\hline Sensitivity & $\begin{array}{c}\text { Ionizing particles } \\
\text { Photons }\end{array}$ & $\begin{array}{c}\text { Ionizing particles } \\
\text { Photons }\end{array}$ & $\begin{array}{l}\text { Off-momentum } \\
\text { electrons }\end{array}$ \\
\hline Bkg limits & Current & Current & Rate \\
\hline Bkg effects & $\begin{array}{c}\text { Detector lifetime } \\
\text { (Trips) }\end{array}$ & $\begin{array}{c}\text { Detector lifetime } \\
\text { (Trips) }\end{array}$ & Radiation damage \\
\hline $\begin{array}{l}\text { MD data } \\
\text { Nov } 95\end{array}$ & $\begin{array}{l}\text { Hit rate radial } \\
\text { in/up/out/down }\end{array}$ & Single hit rate & $\begin{array}{l}\text { Single hit rate } \\
\mathrm{E}>15 \mathrm{GeV}\end{array}$ \\
\hline Info to LEP & TEC1,4,7,10 & "bkg1" & "bkg2" \\
\hline
\end{tabular}

Table B.4: Properties of L3 subdetectors (continued).

\begin{tabular}{|c|c|c|c|}
\hline & VSAT (1996) & ECAL end cap & FB $\mu$-chambers \\
\hline Type & $\begin{array}{c}\text { Very Small Angle Tagger } \\
\text { BGO Calorimeter }\end{array}$ & BGO Calorimeter & Drift chambers \\
\hline Purpose & Tag $2 \gamma$ events & $\begin{array}{c}\text { Calorimeter } \\
\text { e } / \gamma \text { identification }\end{array}$ & Low angle $\mu$ 's \\
\hline Location & $z= \pm 8.1 \mathrm{~m}$ & $z= \pm[0.75-1.0] \mathrm{m}$ & $\begin{array}{c}2.3-5.3 \mathrm{~m} \mathrm{Rad} \\
\mathrm{z}= \pm[5.9-8.3] \mathrm{m}\end{array}$ \\
\hline Size & $4<\theta<10 \mathrm{mrad}$ & $0.74<|\cos (\theta)|<0.98$ & $0.73<|\cos (\theta)|<0.91$ \\
\hline Sensitivity & $\begin{array}{l}\text { Off-momentum } \\
\text { electrons }\end{array}$ & $\begin{array}{l}\text { Off-momentum } \\
\text { electrons }\end{array}$ & Ionizing particles \\
\hline Bkg limits & Singles hit rate & Singles hit rate & Current \\
\hline Bkg effects & Radiation damage & $\begin{array}{l}\text { Radiation damage } \\
\text { Shift energy scale }\end{array}$ & $\begin{array}{l}\text { Detector lifetime } \\
\text { (Trips) }\end{array}$ \\
\hline Info to LEP & & & SUML \& SUMR \\
\hline
\end{tabular}


Table B.5: Properties of DELPHI subdetectors.

\begin{tabular}{|c|c|c|}
\hline & ID (Jet) & Straws (1995) \\
\hline Detector Type & Drift Chamber & Prop.tubes \\
\hline Normal purpose & Tracking,Trigger & Tracking,Trigger \\
\hline $\begin{array}{l}\text { Location,size } \\
\text { Length }\end{array}$ & $\begin{array}{c}r=12-23 \mathrm{~cm} \\
\pm 0.6 \mathrm{~m} \\
24 \text { radial wires } \\
24 \text { sectors }\end{array}$ & $\begin{array}{c}r=23.5-28 \mathrm{~cm} \\
\pm 1.1 \mathrm{~m} \\
5 \text { layers } \\
192 \text { tubes }(\phi)\end{array}$ \\
\hline Operating Cond & $1 \mathrm{Atm} \mathrm{CO}_{2}$-Isobutane & 1 Atm Ar-Ethane \\
\hline Sensitivity & photons $>1 \mathrm{keV}$ & photons $>1 \mathrm{keV}$ \\
\hline Background Limits & current $(\approx 100 \mathrm{nA})$ & trigger rate \\
\hline affected by bkg & detector lifetime & detetor lifetime \\
\hline Background data & $\begin{array}{l}\text { hit rates } \\
\text { radial, angular } \\
\text { distributions. } \\
\text { time distribution }\end{array}$ & $\begin{array}{l}\text { radial, angular } \\
\text { distributions } \\
\text { time distribution }\end{array}$ \\
\hline
\end{tabular}

Table B.6: Properties of DELPHI subdetectors (continued).

\begin{tabular}{|c|c|c|c|}
\hline & $\mathrm{TPC}$ & STIC & VSAT \\
\hline Type & TPC & $\mathrm{Pb} /$ Scint & $\begin{array}{c}\mathrm{W} / \mathrm{Si} \\
\text { calorimeter }\end{array}$ \\
\hline Purpose & Tracking & Luminosity & Luminosity \\
\hline Radial size & $0.35-1.11 \mathrm{~m}$ & $\begin{array}{c}65-418 \mathrm{~mm} \\
29.5-188 \mathrm{mrad}\end{array}$ & $\theta=5-7 \mathrm{mrad}$ \\
\hline $\begin{array}{l}\text { Length } \\
\text { Location }\end{array}$ & $\begin{array}{c}\phi 12 \text { sectors } \\
\pm 1.34 \mathrm{~m} \\
\text { IP }\end{array}$ & $z= \pm 2.20 \mathrm{~m}$ & $\begin{array}{l}\phi 180 \mathrm{mrad} \\
z= \pm 7.70 \mathrm{~m}\end{array}$ \\
\hline Operating Cond & Ar/Methane, $1 \mathrm{Atm}$ & & \\
\hline Sensitivity & $\begin{array}{l}\text { photons } \\
\gtrsim 10 \mathrm{keV}\end{array}$ & $\begin{array}{l}\text { electrons } \\
\gtrsim 10 \mathrm{GeV}\end{array}$ & $\begin{array}{l}\text { electrons } \\
\gtrsim 20 \mathrm{GeV}\end{array}$ \\
\hline Bkg Limits & & trigger rate & \\
\hline $\begin{array}{l}\text { Affected by } \\
\text { background }\end{array}$ & Ageing, resolution & trigger rate & $\begin{array}{l}\text { luminosity } \\
\text { precision }\end{array}$ \\
\hline $\begin{array}{l}\text { background } \\
\text { data }\end{array}$ & $\begin{array}{l}\text { Ave. sector rate } \\
\text { photon hits } \\
\text { "bkg1" = hit rate }\end{array}$ & $\begin{array}{c}\text { spatial, energy } \\
\text { distributions }\end{array}$ & $\begin{array}{l}\text { energy, rates } \\
\text { "bkg2" = hit rate }\end{array}$ \\
\hline
\end{tabular}


Table B.7: Properties of Background Monitoring Detectors.

\begin{tabular}{|c|c|c|c|}
\hline & $\begin{array}{c}\text { ALEPH } \\
\text { Small Angle } \\
\text { Bkg monitor }\end{array}$ & $\begin{array}{l}\text { ALEPH } \\
\text { Radiation } \\
\text { Monitor }\end{array}$ & $\begin{array}{l}\text { DELPHI } \\
\text { TPC } \\
\text { Monitor }\end{array}$ \\
\hline Type & Drift Chamber & Photodiodes & Si pads \\
\hline Purpose & robust & $\begin{array}{c}\text { Monitor } \\
\text { Vertex detector }\end{array}$ & Monitor \\
\hline Location,size & $\begin{array}{c}\theta=26-63 \mathrm{mrad} \\
64-147 \mathrm{~mm} \\
z= \pm 2.30 \mathrm{~m}\end{array}$ & $\begin{array}{l}4 \times 100 \mathrm{~mm}^{2} \\
z=360 \mathrm{~mm}\end{array}$ & $\begin{array}{c}6 \times 40 \mathrm{~mm}^{2} \\
\text { at } 0.3 \mathrm{~m} \text { radius } \\
z=1.90 \mathrm{~m}\end{array}$ \\
\hline Operating Cond & $1 \mathrm{Atm}$ Ar- $\mathrm{CO}_{2}$ & & \\
\hline Sensitivity & $\begin{array}{c}\text { photons } \gtrsim 10 \mathrm{keV} \\
\text { electrons }\end{array}$ & photons $\gtrsim 10 \mathrm{keV}$ & \\
\hline $\begin{array}{l}\text { Background } \\
\text { data }\end{array}$ & $\begin{array}{c}\text { hit rate } \\
\text { time distributions } \\
\text { far sources } \lesssim 120 \text { m } \\
\text { "bkg2"=rate }\end{array}$ & $\begin{array}{l}\text { instantaneous, } \\
\text { intgerated } \\
\text { dose }\end{array}$ & $\begin{array}{c}\text { photon energy } \\
\text { photon time }\end{array}$ \\
\hline
\end{tabular}

Table B.8: Properties of Background Monitoring Detectors (continued).

\begin{tabular}{|c|c|c|}
\hline & $\begin{array}{l}\text { OPAL } \\
\text { Background } \\
\text { Monitor }\end{array}$ & $\begin{array}{l}\text { OPAL } \\
\text { Radiation } \\
\text { Monitor }\end{array}$ \\
\hline Type & Proportional tubes & Silicon Diodes \\
\hline Purpose & robust & $\begin{array}{c}\text { Monitor } \\
\text { Vertex detector }\end{array}$ \\
\hline Location,size & $\begin{array}{c}65 \mathrm{~mm} \times 10 \mathrm{~mm} \text { diam } \\
r=0.22 \mathrm{~m} \\
z= \pm 1.0 \mathrm{~m}\end{array}$ & $\begin{array}{c}8 \times 8 \mathrm{~mm} \\
r=70 \mathrm{~mm} \\
z= \pm 0.3 \mathrm{~m}\end{array}$ \\
\hline Operating Cond & $4 \mathrm{Atm} \mathrm{Ar}-\mathrm{CH}_{4}-\mathrm{iC}_{4} \mathrm{H}_{10}$ & \\
\hline Sensitivity & $\begin{array}{c}\text { photons } \gtrsim 10 \mathrm{keV} \\
\text { electrons }\end{array}$ & electrons \\
\hline Background data & hit rates & radiation level \\
\hline
\end{tabular}

